\title{
Multiparametric measurement of cerebral physiology using calibrated fMRI
}

\author{
Molly G Bright ${ }^{1,2}$, Paula L Croal ${ }^{3}$, Nicholas P Blockley ${ }^{4}$, Daniel P Bulte ${ }^{3,4}$
}

1. Sir Peter Mansfield Imaging Centre, School of Medicine, University of Nottingham, Nottingham, UK.

2. Department of Physical Therapy and Human Movement Sciences, Feinberg School of Medicine, Northwestern University, Chicago, IL.

3. IBME, Department of Engineering Science, University of Oxford, Oxford, UK.

4. FMRIB, Nuffield Department of Clinical Neurosciences, University of Oxford, Oxford, UK.

\begin{abstract}
The ultimate goal of calibrated $\mathrm{fMRI}$ is the quantitative imaging of oxygen metabolism $\left(\mathrm{CMRO}_{2}\right)$, and this has been the focus of numerous methods and approaches. However, one underappreciated aspect of this quest is that in the drive to measure $\mathrm{CMRO}_{2}$, many other physiological parameters of interest are often acquired along the way. This can significantly increase the value of the dataset, providing greater information that is clinically relevant, or detail that can disambiguate the cause of signal variations. This can also be somewhat of a double-edged sword: calibrated fMRI experiments combine multiple parameters into a physiological model that requires multiple steps, thereby providing more opportunity for error propagation and increasing the noise and error of the final derived values. As with all measurements, there is a trade-off between imaging time, spatial resolution, coverage, and accuracy. In this review, we provide a brief overview of the benefits and pitfalls of extracting multiparametric measurements of cerebral physiology through calibrated fMRI experiments.
\end{abstract}

Key words: $\quad B O L D, F M R I, C B F, A S L$, gas, calibration

Word count: 12,656

Contact: Prof. Daniel Bulte

Institute of Biomedical Engineering

Department of Engineering Science

Old Road Campus Research Building

University of Oxford

Headington

Oxford OX3 7DQ, UK

daniel.bulte@eng.ox.ac.uk 
+441865617300

Abbreviations

AAT

AD

ASL

BOLD

CBF

CBV

$\mathrm{CMRO}_{2}$

CT

CVR

$\mathrm{dHb}$

DOT

DSC

EEG

EPI

$\mathrm{FiCO}_{2}$

$\mathrm{FiO}_{2}$

fMRI

$\mathrm{Hb}$

Hct

LDF

MEG

OEF

$\mathrm{PaO}_{2}$

$\mathrm{PaCO}_{2}$

pCASL

PET

$\mathrm{P}_{\mathrm{ET}} \mathrm{O}_{2}$

$\mathrm{PETCO}_{2}$

PLD

$\mathrm{PO}_{2}$

$\mathrm{SaO}_{2}$

SNR

SPECT

$\mathrm{SvO}_{2}$

TCD
Arterial arrival time

Alzheimer's disease

Arterial spin labelling

Blood oxygenation level dependent

Cerebral blood flow

Cerebral blood volume

Cerebral metabolic rate of oxygen

Computed tomography

Cerebrovascular reactivity

Deoxyhaemoglobin

Diffuse Optical Tomography

Dynamic susceptibility contrast

Electroencephalography

Echo planar imaging

Inspired fraction of carbon dioxide

Inspired fraction of oxygen

Functional magnetic resonance imaging

Haemoglobin

Haematocrit

Laser Doppler Flowmetry

Magnetoencephalography

Oxygen extraction fraction

Arterial partial pressure of oxygen

Arterial partial pressure of carbon dioxide

Pseudo-continuous arterial spin labelling

Positron emission tomography

End tidal partial pressure of oxygen

End tidal partial pressure of carbon dioxide

Post-label delay

Partial pressure of oxygen

Arterial saturation of oxygen

Signal to noise ratio

Single photon emission tomography

Venous saturation of oxygen

Transcranial Doppler ultrasound 
TRUST $\quad \mathrm{T}_{2}$ Relaxation Under Spin Tagging

VASO Vascular space occupancy

vCBV Venous cerebral blood volume

VERVE Venous refocusing for volume estimation

\section{Introduction}

Functional MRI is dominated by blood oxygenation level dependent (BOLD) imaging of changes in the gradient echo signal during a task or stimulus, which provides robust sensitivity to local haemodynamic changes and can be easily implemented in most clinical and research scanners. It has proven to be a powerful tool for localising activity in the brain, and has revolutionised neuroimaging (Bandettini, 2012). Despite the physiological origins of the BOLD signal, it is not a pure or quantitative signal; BOLD contrast is driven by relative changes in more than one physiological parameter, and thus dependent on both baseline physiology and the magnitude of any neurovascular changes. If we wish to know more than simply the location of functional activity in the brain, to compare activation between sessions, subjects, scanners or sites, or to account for the effects of interventions on brain function, then BOLD fMRI alone is not sufficient. To address these aims, we can employ additional imaging contrasts and models of human physiology and MRI physics to "calibrate" the BOLD fMRI signal, resulting in a meaningful physiological parameter that reflects specific aspects of brain function in health or disease.

The ways in which such calibration experiments have been implemented over the last 20 years have evolved (see article by Gauthier et al. in this issue), but the goal of obtaining quantitative physiological images remains the same, primarily focussing on the quantification of changes in the cerebral metabolic rate of oxygen consumption $\left(\mathrm{CMRO}_{2}\right)$. Importantly, all variants require the collection of additional imaging data beyond BOLD-weighted scans, which may provide valuable insight into physiological processes by themselves, or through combined modelling. In this way, calibrated fMRI experiments provide a framework for extended multiparametric physiological imaging. Rather than only considering the endpoint of the calibration process, i.e. $\mathrm{CMRO}_{2}$, the extra effort required for acquiring and analysing a calibrated fMRI dataset can be justified by the added value of the numerous distinct physiological parameters that are extracted. A compound parameter such as the BOLD signal is difficult to interpret when dealing with patients, subjects with comorbidities, different treatment arms in clinical trials, pharmacological interventions, and natural variations in 
physiology between individuals. By obtaining a richer dataset along the road to calibration, there is a greater chance of determining the mechanisms of action in disease and comparing like-for-like in specific patient cohorts, thus enabling more complex questions to be considered.

In this paper, we describe each parameter that can be acquired or derived in a calibrated fMRI experiment designed either to provide a relative change in a physiological parameter during some period of elevated brain activity, or a baseline value at rest. The gas challenges utilised for calibration have direct, unintended consequences on the imaging data, and it is important to adjust scanning and analysis protocols to minimise or account for these effects. However, despite these challenges, the resulting diversity in physiological information adds immensely to our understanding of brain function and neurological disease.

\section{Calibrated fMRI}

The most successful approach for modelling the BOLD signal in terms of physiological parameters was first presented by Davis et al. (1998), and later refined by Hoge et al. (1999). These models express a BOLD signal change in response to a stimulus or task in terms of relative changes in cerebral blood flow (CBF), cerebral blood volume (CBV) and $\mathrm{CMRO}_{2}$. Written out in its full form, and shown schematically in Figure 1, the BOLD signal can be shown to be a function of these haemodynamic and metabolic changes scaled by $M$, the BOLD calibration constant.

$$
\frac{\triangle B O L D}{B O L D_{0}}=M\left(1-\left(\frac{C B V}{C B V_{0}}\right)\left(\frac{C B F}{C B F_{0}}\right)^{-\beta}\left(\frac{C M R O_{2}}{C M R O_{2,0}}\right)^{\beta}\right)
$$

Here, physiological parameters during the active or stimulated state (e.g., CBV) are normalised by their value at baseline (e.g., $C B V_{0}$ ). The exponent $\beta$ controls for the effect of vessel size on the acquired data (Ogawa et al., 1993). Section 4.3 details the origin of $\beta$ and the appropriate selection of its value. As discussed in greater detail in subsequent sections, CBV changes are rarely imaged directly in calibrated fMRI; instead they are inferred from changes in CBF, which is more easily measured using Arterial Spin Labeling (ASL) techniques (see section 3.2). This interaction between CBF and CBV (as shown in Figure 1) is described by a model of CBF-CBV coupling and characterised by the constant $\alpha$, selection of which is covered in section 4.2 . 


$$
\frac{\triangle B O L D}{B O L D_{0}}=M\left(1-\left(\frac{C B F}{C B F_{0}}\right)^{\alpha-\beta}\left(\frac{C M R O_{2}}{C M R O_{2,0}}\right)^{\beta}\right)
$$

In order to estimate changes in $\mathrm{CMRO}_{2}$, measurements of the changes in the BOLD signal and CBF are required, as well as the BOLD calibration constant, $M$. Unlike BOLD and CBF, $M$ is not directly measured, rather it is estimated through a calibration experiment. This experiment involves the administration of air with either added carbon dioxide $\left(\mathrm{CO}_{2}\right)$ or an increased oxygen $\left(\mathrm{O}_{2}\right)$ content, and is described in further detail in the next section. However, by acquiring these additional measurements it is possible to extract specific physiological information (namely $\mathrm{CMRO}_{2}$ ) from the otherwise complex haemodynamic and metabolic contributions to the BOLD signal.

Calibrated fMRI has historically focussed on the measurement of relative changes in $\mathrm{CMRO}_{2}$ due to brain activity. However, more recently this technique has been extended to baseline measurements of $\mathrm{CMRO}_{2}$, reflecting the activity of the brain at rest. This new group of techniques was developed nearly simultaneously by three research groups (Bulte et al., 2012; Gauthier and Hoge, 2012a; Wise et al., 2013). In contrast to conventional calibrated fMRI, these techniques aim to separate the contributions to $M$, rather than just account for its effect on the BOLD signal (see Figure 1). By considering the derivation of Eq. (1), the sensitivity of the BOLD signal to baseline blood oxygenation (or more specifically deoxygenation) can be observed.

$$
\frac{\Delta B O L D}{B O L D_{0}}=M\left(1-\left(\frac{C B V}{C B V_{0}}\right)\left(1+\frac{\Delta[d H b]}{[d H b]_{0}}\right)^{\beta}\right)
$$

Here, $[\mathrm{dHb}]_{0}$ is the concentration of deoxyhaemoglobin $(\mathrm{dHb})$ in venous blood vessels at rest and $\Delta[\mathrm{dHb}]$ is the change in $\mathrm{dHb}$ concentration during the calibration step. Since $[\mathrm{dHb}]_{0}$ is a function of venous blood oxygen saturation, it can be used to calculate the oxygen extraction fraction (OEF) and, in combination with baseline $\mathrm{CBF}$, baseline $\mathrm{CMRO}_{2}$ can be calculated. However, we are now left with two unknowns; $M$ and $\Delta[\mathrm{dHb}]$. The solution to this problem is to perform two calibration experiments: the first to estimate $M$ and the second where $\Delta[\mathrm{dHb}]$ can be modelled accurately by using a stimulus that has a well characterised effect on blood oxygenation. Therefore, we will refer to this group of techniques for measuring baseline OEF and $\mathrm{CMRO}_{2}$ as dual calibrated $\mathrm{fMRI}$.

The calibration experiment is important to both conventional calibrated $\mathrm{fMRI}$ and dual calibrated fMRI. The stimulus for this calibration generally consists of gas inhalation and will 
be considered in the following section. There is also the prospect of gas free calibration, which is considered in section 7.2.

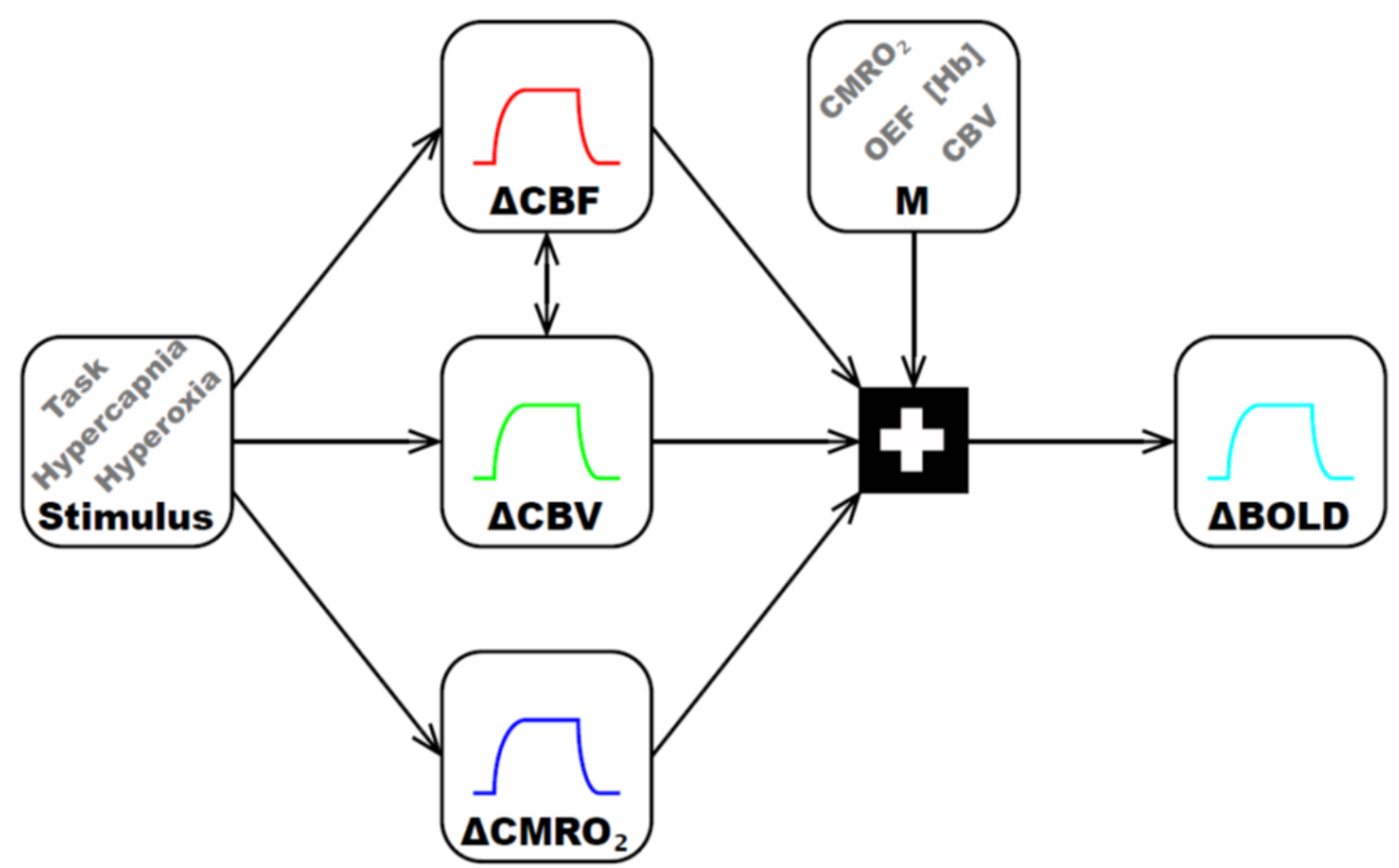

Figure 1. The Davis model describes the major physiological drivers of changes in the BOLD signal. BOLD signal changes can be driven by stimulus-related changes in CBF, CBV and $\mathrm{CMRO}_{2}$. Gas stimuli, such as a hypercapnia challenge, may impact CBF and CBV without altering baseline $\mathrm{CMRO}_{2}$, and additional imaging data can be acquired to estimate these dynamic changes in CBF, CBV, or both. However, knowledge of changes in haemodynamics and oxygen metabolism are not sufficient to describe the magnitude of the BOLD signal change: baseline physiological parameters such as OEF and CBV determine the maximum BOLD signal change and hence the scaling of the BOLD response (via the BOLD calibration constant, $M$ ).

\subsection{Calibration via gas inhalation challenges}

Gas inhalation has been used since the earliest days of fMRI to normalise the BOLD signal (Bandettini and Wong, 1997). Initially this consisted of a brief period of hypercapnia via an increased inspired fraction of $\mathrm{CO}_{2}$. This gas challenge was used to induce an isometabolic increase in $\mathrm{CBF}$, thereby reducing OEF and increasing the BOLD signal. It remains controversial whether gas challenges can be considered truly isometabolic in these experiments (see Section 4.5 for further discussion). However, by making this assumption, 
Eq. 2 can be used to estimate $M$ when combined with measurements of CBF and BOLD (see section 4.4 for details).

Following the same principle, hypercapnia calibration was later followed by hyperoxia calibration, which is induced by administration of air with an increased $\mathrm{O}_{2}$ fraction. By assuming isometabolism, Eq. 3 can be used to estimate M by combining CBV (inferred from CBF measurements) and BOLD data with oxygen transport modelling to estimate $\Delta[\mathrm{dHb}]$ (Chiarelli et al., 2007). Similarly to hypercapnia, there has been some controversy over whether hyperoxia is truly isometabolic (see Section 4.5). Further details on the implementation of hyperoxia calibration can be found in Section 4.4.

Administration of hypercapnic and hyperoxic gas mixtures follow common approaches. The earliest, and most simplistic, approaches utilised a Douglas bag pre-filled with a fixed gas content (known as a fixed inspired challenge), which was switched with room air using a manifold and inhaled through a closed breathing mask (Douglas and Haldane, 1905). These techniques have been supplemented by potentially more accurate and repeatable (Fisher, 2016) automated gas delivery systems based on feed-forward (Prisman et al., 2008) or feedback algorithms (Wise et al., 2007). Choice of gas delivery should be informed by budgetary constraints, patient comfort and stimulus accuracy and repeatability requirements.

\section{Acquired measurements}

\subsection{BOLD}

As indicated in Figure 1, the BOLD signal is the product of complex and coupled changes in multiple vascular and metabolic factors, and can be additionally modulated via pulse sequence parameters used in scanning. As such, the baseline BOLD-weighted signal is without direct physiological interpretation. However, BOLD fMRI, where changes in BOLD-weighted signals are sampled, remains the dominant workhorse of the neuroimaging field for good reasons: it has high SNR, is extremely sensitive to changes in oxygenation, can be sampled with rapid, high-resolution acquisition schemes, and ultimately allows for exquisite characterisation of subtle changes in brain activity.

Whilst the BOLD effect was first demonstrated in 1990 (Ogawa et al., 1990), the background to understanding its origin had largely already been discovered. NMR experiments in the 1980 's revealed that the transverse relaxation time, $T_{2}$, of blood was oxygenation dependent 
(Thulborn et al., 1982). Hence, the increased blood oxygenation observed by Ogawa and colleagues in their hypercapnia experiments resulted in an increase in $T_{2}$ and the disappearance of the venous blood vessels that had appeared as dark lines during air breathing. However, this intravascular effect is dependent on the blood volume, which makes up only a small fraction of the tissue volume, and so a second mechanism was hypothesised to contribute to the blooming of the venous vessels. This mechanism is based on the magnetic susceptibility of haemoglobin, which was discovered in 1936 (Pauling and Coryell, 1936). Importantly, it was the compartmentalisation of this susceptibility within venous blood vessels, and its relative paramagnetism with respect to the surrounding tissue, that resulted in an extravascular contribution to the BOLD effect (Ogawa et al., 1993). Protons in the tissue surrounding deoxygenated blood vessels experience the magnetic field gradients generated by this susceptibility difference resulting in dephasing of the magnetisation and an enhancement of the transverse relaxation time known as $\mathrm{T}_{2}{ }^{*}$.

Despite this explanation of the BOLD effect, it wasn't entirely clear that this would be useful for mapping brain activity. It is widely known that $\mathrm{CBF}$ and $\mathrm{CMRO}_{2}$ are tightly coupled during baseline and it was anticipated that during increased brain activity this would be maintained. Under these conditions, blood oxygenation would be unchanged and the BOLD effect would be blind to changes in brain activity. Fortunately, results from Positron Emission Tomography (PET) had revealed a focal uncoupling of $\mathrm{CBF}$ and $\mathrm{CMRO}_{2}$ during sensory stimulation in the years preceding the discovery of the BOLD effect (Fox and Raichle, 1986). The implication of this is that during focal brain activity changes in $\mathrm{CBF}$ exceed changes in $\mathrm{CMRO}_{2}$, resulting in a decreased OEF, increased blood oxygenation and an increase in the BOLD signal. However, as Eq. 3 makes clear, the changes in the BOLD signal do not result purely from changes in the blood oxygenation (described as changes in [dHb]), but also depend on changes in CBV. As such, changes in the BOLD signal can be considered proportional to the absolute change in $\mathrm{dHb}$ content and $M$ as being proportional to the $\mathrm{dHb}$ content of the voxel at rest.

When BOLD contrast was first explored in the early 1990's, echo planar imaging (EPI) (Mansfield, 1977) enabled rapid acquisition of $\mathrm{T}_{2}{ }^{*}$-weighted contrast, achieving temporal resolutions sufficient to resolve functional changes in blood oxygenation (2-3.5 s) (Bandettini et al., 1992; Belliveau et al., 1992; Kwong et al., 1992). Most BOLD fMRI experiments today follow very similar practices to these early studies, although higher field strengths, advances in scanner hardware, new acceleration techniques, and novel read-out strategies have improved data quality, enabling faster scanning of higher resolution data with better stability, and higher SNR and CNR. For example, large multi-centre initiatives, such as the Human Connectome Project, routinely collect BOLD-weighted fMRI data using multiband pulse 
sequences to achieve whole-brain coverage with $2 \mathrm{~mm}$ isotropic voxel resolution in just $700 \mathrm{~ms}$ (Van Essen et al., 2013). 3D-EPI techniques may achieve similar resolutions with even higher SNR (Stirnberg et al., 2017). It remains to be seen how these exciting advances in BOLDweighted acquisition techniques, which are being adopted widely across the fMRI community, will be implemented in the context of calibrated fMRI.

\section{$3.2 \quad C B F$}

Since the original work by Davis et al. (1998) the most prevalent secondary measurement used to calibrate the BOLD signal has been CBF. This was originally a measure of the relative change in CBF due to a hypercapnic stimulus using a Flow-Sensitive Alternating Inversion Recovery (FAIR) spin echo pulsed arterial spin labelling (PASL) sequence in a separate scan, but was rapidly advanced to an interleaved technique to enable simultaneous measurement of the BOLD and flow responses (Hoge et al., 1999).

As advances in CBF imaging using MRI were made, these were incorporated into calibration methods in order to improve coverage, resolution, speed and accuracy, while the BOLD imaging remained essentially unchanged. Initially this was by replacing the FAIR sequence with QUIPSS II (Wong et al., 1998) or Q2TIPS (Luh et al., 1999) in order to reduce errors due to transit time delays, and also enable a degree of absolute flow quantification. The technique also moved from single slices to multi-slice images, initially 5 to 8 slices at 5 to $7 \mathrm{~mm}$ thick. This heralded somewhat of a golden age for calibrated fMRI. Interleaved sequences were replaced with dual-echo ones to dramatically increase temporal resolution and thus improve fitting statistics. Later, image read-out options were further expanded as the dual-echo approach was refined to the double excitation (DEXI) method, which improved the SNR for combined BOLD and ASL approaches, and reduced cross-contamination of the signals (Schmithorst et al., 2014).

The rise in popularity of pseudo-continuous ASL (pCASL) sequences was a watershed moment for ASL (Dai et al., 2008); these sequences offered an increase in SNR that enabled higher spatial resolution and greater brain coverage. Techniques for acquiring PCASL data with multiple post-label delays (PLDs) allowed more accurate quantitative CBF maps using the Buxton model (Richard B Buxton et al., 1998), comparing favourably to ${ }^{15}$ O-PET CBF measurements (Zhang et al., 2014), and facilitating the estimation of regional arterial arrival time (AAT) changes during hypercapnia. Initially greeted with enthusiasm by those seeking calibration, there quickly came a note of warning: although pCASL was apparently superior 
for quantitative measurement of resting CBF or changes in CBF during sensory stimuli or cognitive tasks, an additional (and important) confound occurs during calibrated fMRI with gas challenges. pCASL sequences rely on inverting spins in a thin tagging plane below the imaging volume. Due to thermodynamics, and as the spins are moving through this plane with varying velocities during the cardiac cycle, the efficiency of this inversion is not $100 \%$. It is generally assumed that the inversion efficiency is approximately 85-95\% (Juttukonda et al., 2017; Mezue et al., 2014). The effect of a global vasodilatory stimulus such as hypercapnia is a significant increase in the flow velocity through the major feeding arteries such as the internal carotid and vertebral arteries. This results in a significant reduction in the inversion efficiency (Aslan et al., 2010), which would need to be measured and taken into account in order to maintain accuracy when comparing pCASL results during hypercapnia and at baseline (Chen et al., 2017). Furthermore, the inversion efficiency is unlikely to be the same in each of the four main neck arteries, as the carotids are generally wider than the vertebrals, and there is often a significant disparity in diameter between the two vertebrals themselves. The relative change in inversion efficiency during gas challenges is thus also likely to vary between arteries, impacting the CBF values measured in their respective territories (Berry et al., 2015). Flow velocity in large arteries may also influence the regional AAT of the labelled blood in downstream tissue.

There are multiple variants of the pCASL sequence that have their own intrinsic behaviours, and are impacted differently by the choice of sequence parameters, labelling planes, and gas stimuli. Fundamentally, a pCASL sequence may be either balanced or unbalanced, which can have a significant impact on the labelling efficiency due to $B_{0}$ inhomogeneity (Jahanian et al., 2011; Wu et al., 2007). Numerous labelling and read-out parameters may vary between pCASL implementations, including the labelling pulse shape, mean labelling gradient, maximum labelling gradient, labelling flip angle, labelling duration, initial post-label delay, labelling position, readout approach, number of slices, slice thickness, matrix size, field of view, fat suppression, parallel imaging, B1-filtering, partial Fourier, background suppression, vascular suppression, and number of label-control pairs (Mutsaerts et al., 2015). There are steps that can be taken to minimise the impact of the choice of pCASL sequence and parameters on the CBF measurement (Zhao et al., 2016), and care must be taken with these. A recent multi-vendor study testing reproducibility noted: "Small differences in sequence parameters can have a larger effect on the reproducibility of ASL than hardware or software differences between vendors." (Mutsaerts et al., 2015).

In light of these issues, a consensus has yet to be formed as to which is the optimal approach for measuring changes in CBF for calibration purposes. Considering the sensitivity of pCASL 
to arterial flow velocities and arrival times, it may be that although pCASL is superior for measuring CBF in general, it is PASL, with its relative insensitivity to bulk changes in flow within the labelling region, which is a better choice for calibration.

\subsection{CBV}

Whilst CBF is the most common secondary measurement used to calibrate the BOLD response, it is also important to account for the effect of stimulus induced changes in CBV. In conventional calibrated fMRI, following the Davis model (described in Section 2), this is achieved by using a measurement of the change in CBF (from an ASL acquisition) as a proxy for changes in CBV. The relationship between these parameters can be described by the wellestablished power law relationship $\left(\mathrm{CBV} \propto \mathrm{CBF}^{\alpha}\right)$, where the exponent is commonly known as the Grubb constant (Grubb et al., 1974). In this work, a value of $\alpha=0.38$ was measured for the change in CBV across all vascular compartments (arterial, capillary and venous), otherwise known as total CBV (See Section 4.2 for more detailed discussion of $\alpha$ ).

However, in order to accurately model the effect of changes in CBV on the BOLD response, it is important to understand the physiology that underlies BOLD contrast. In the early days of (uncalibrated) BOLD fMRI it was assumed that the majority of the change in CBV during "activation" was localised to the venous blood vessels (Buxton et al., 1998; Mandeville et al., 1999). However, it became clear that this was not the case, and that the change in CBV is much larger in arterial vessels (Lee et al., 2001). For healthy human subjects, blood within the arterial vessels has an oxygen saturation approaching $100 \%$. As the BOLD response is largely dependent on changes in the deoxyhaemoglobin content of the voxel, we do not anticipate a meaningful contribution to the BOLD signal from arterial vessels. Therefore, the relevant change in CBV for calibrated $\mathrm{fMRI}$ is not the large arterial dilation, but rather the small fraction of the total CBV change that originates from the veins and capillaries. Incorporating this understanding of the physiology into the acquisition and analysis of calibrated fMRI data is important to minimise systematic error, and techniques to measure the change in venous $\mathrm{CBV}$ are required to enable more accurate estimation of $\alpha$, or to incorporate directly in the estimation of $\mathrm{CMRO}_{2}$.

One such method, known as the venous refocusing for volume estimation (VERVE) technique, specifically measures changes in venous CBV (Stefanovic and Pike, 2005). The VERVE technique works by applying a train of refocusing pulses to recover signal lost to the dephasing effect of deoxygenated blood. Venous blood can be isolated by altering the inter-pulse spacing, which results in additional signal loss in deoxygenated blood but leaves oxygenated 
blood unaffected. By combining measurements of venous CBV from VERVE with ASL-based measurements of CBF during an fMRI stimulus paradigm, a venous specific value of $\alpha$ of 0.23 has been estimated (Chen and Pike, 2009), which is now routinely used in calibrated fMRI experiments (Bulte et al., 2012; Gauthier and Hoge, 2012a). Unfortunately, VERVE is limited to a single slice acquisition, making it impractical as a means to measure venous CBV in the more general application of calibrated fMRI. However, by refining the estimation of $\alpha$, this technique has played an important role in reducing systematic error in the estimation of stimulus-evoked changes in $\mathrm{CMRO}_{2}$.

The simultaneous acquisition of changes in BOLD, CBF and CBV has been achieved by using the vascular space occupancy (VASO) method (Lin et al., 2008). This has the advantage that the resulting estimates of CBV are subject and stimulus specific, which may be important in applications where CBF-CBV coupling is believed to be altered (See Section 4.2 for further detail). The VASO technique relies on the differing longitudinal relaxation times, $T_{1}$, between tissue and blood. By applying a global inversion pulse and imaging at the inversion time, $\mathrm{TI}$, at which the blood signal is nulled, the signal can be sensitised to CBV. Interestingly, since this involves signal nulling, increases in CBV translate to decreases in the VASO signal, as a greater volume of blood signal is nulled. Unfortunately, there are two disadvantages to the VASO technique. Firstly, unlike ASL, the ratio of the activated condition to the baseline condition does not give a measure of the fractional change in CBV; baseline CBV must be known or assumed (Lu et al., 2003). Secondly, by inverting the whole blood volume, VASO is sensitive to total CBV rather than the venous CBV that underlies BOLD contrast. Therefore, it is likely to overestimate the contribution of CBV changes, which will propagate into an underestimation of changes in $\mathrm{CMRO}_{2}$.

The estimation of venous CBV using hyperoxia follows the principles of dynamic susceptibility contrast (DSC) MRI; the usual Gadolinium-based contrast agent is effectively replaced by deoxygenated haemoglobin acting as a non-diffusible tracer. By increasing the oxygen content of arterial blood, largely through an increase in the oxygen content of plasma, the oxygen saturation of venous blood is increased. In DSC MRI, absolute CBV is measured by normalising signals to a voxel containing only arterial blood, referred to as the arterial input function (AIF). However, the signal from arterial blood is not affected by hyperoxia due to the oxygenation saturation being nearly $100 \%$. Instead, the signal from the sagittal sinus (isolating a voxel containing only venous blood) can be used to perform a similar normalisation (Bulte et al., 2007b). This normalisation step aims to account for the true concentration of the contrast agent (or, in this case, $\mathrm{dHb}$ content) in the blood. A blood sample could be drawn and analysed for either gadolinium concentration or oxygen saturation, but this is typically undesirable due 
to the invasiveness and time-intensive nature of such a procedure. Fortunately, with a gas challenge such as hyperoxia, the effect on arterial gas tensions can be estimated by measuring the oxygen content of the subject's expired breaths (as described in Section 4.4), enabling the change in arterial saturation due to the gas challenge to be calculated (Severinghaus, 1979). It has since been shown that the hyperoxia BOLD signal change is linearly dependent on $\mathrm{PaO}_{2}$ (Blockley et al., 2013). Therefore, maps of the hyperoxia BOLD signal change represent relative venous $\mathrm{CBV}$ at rest, with absolute maps generated by appropriate scaling. This scale factor can be calculated using a heuristic model derived from detailed simulations of the BOLD signal, which is a function of echo time and $\mathrm{PaO}_{2}$. A similar approach can be used to estimate changes in venous CBV (Blockley et al., 2012a).

The use of the hyperoxia BOLD signal to measure venous CBV may seem inconsistent with its use to calibrate the BOLD response (Chiarelli et al., 2007). However, hyperoxia-based calibrated fMRI requires the baseline OEF to be assumed. Whilst this may be considered a limitation in the context of measuring stimulus evoked changes in $\mathrm{CMRO}_{2}$, this unknown is what enables dual calibrated fMRI to measure OEF. Intuitively, this can be explained as varying the value of OEF until the estimates of $M$ are consistent between hypercapnia and hyperoxia (Gauthier and Hoge, 2012a) or by dividing out the effect of venous CBV from the hypercapnia $M$ value (Blockley et al., 2015b).

\subsection{End-tidal gases}

Key to understanding the influence of the gas challenges is real-time monitoring of the participant's end-tidal gas levels. End-tidal values represent the averaging of alveolar gas levels, and are used to represent our best approximation of $\mathrm{PaO}_{2}$ and $\mathrm{PaCO}_{2}$. As $\mathrm{O}_{2}$ and $\mathrm{CO}_{2}$ diffuse rapidly across biological membranes, arterial partial pressures rapidly reach a steady state with alveolar partial pressures. However, the two spaces do not have equal partial pressures (i.e. $\mathrm{PaO}_{2} \neq \mathrm{P}_{\mathrm{ET}} \mathrm{O}_{2}$ ) due to the alveolar-arterial gradient (A-a gradient), which effectively represents the (in)efficiency of the lung surface. However, changes in end-tidal levels are linearly correlated with changes in arterial partial pressures. Several studies have demonstrated tight coupling between end-tidal and arterial values, although systematic biases have been reported that can be further altered by exercise or aging (Peebles et al., 2007; Robbins et al., 1990; Young et al., 1991). Certain methods for administering and monitoring gas challenges may make this relationship more accurate (Ito et al., 2008). In practice, endtidal values are a pragmatic and robust approach for monitoring the effects of a hypercapnic or hyperoxic gas challenge during scanning. 


\section{Assumptions, error, and variability}

Here we consider the compromises necessary when acquiring multiple contrasts (i.e. BOLD and $\mathrm{CBF}$ weighting) in one imaging session. We examine the meaning and derivation of model parameters $\alpha$ and $\beta$, the values typically assumed, and methods for empirically determining them. The BOLD scaling factor, $M$, is described, paying additional attention to the error and variability that can occur due to incorrect assumptions regarding haematocrit (Hct) and arterial oxygen saturation $\left(\mathrm{SaO}_{2}\right)$. Finally, the Davis model assumes that the gas challenges used in calibration are isometabolic (do not alter $\mathrm{CMRO}_{2}$ ); we present the controversial findings in the literature regarding this fundamental assumption.

\subsection{Compromises in scan acquisition}

The requirement to obtain multiple, distinct image contrasts concurrently in one scan inevitably results in less optimal individual images than if each were collected in isolation. Trade-offs are necessary, selecting temporal resolution, spatial resolution, voxel size, brain coverage, and echo time values that enable sufficient SNR across all acquired image parameters. Several examples of concurrent acquisition techniques have already been described above.

Obtaining BOLD signal by adding together ASL image pairs requires using a TE that is suboptimal for either BOLD, ASL, or both. Interleaving ASL and BOLD acquisitions has very poor temporal resolution, due to the post-label delay of the ASL acquisition and need for pairs of labeled and control images, resulting in very few data points during short stimuli. In combined sequences, the inherently low SNR of ASL necessitates lower spatial resolution to achieve enough signal to quantify CBF. In dual-echo ASL-BOLD acquisitions, an early echo time is used to maximise sensitivity to a decaying label of blood, and a later echo time is selected to achieve greater BOLD-contrast weighting. Collecting data at 2 or more echo times places practical limits on when these echoes can occur and on the number of data points acquired in that time period (either in terms of voxel resolution or brain coverage). Both echo times are usually suboptimal, and both spatial resolution and brain coverage are less than could be obtained with a simple BOLD sequence. There is also cross-talk between the two datasets in a dual-echo approach: BOLD changes can contaminate the first CBF-weighted echo, and ASL tagging can remain in the second BOLD-weighted echo. This can be greatly mitigated by using a DEXI sequence instead (Schmithorst et al., 2014). Collecting 
simultaneous BOLD data and VASO estimates of CBV encounters similar concerns, as VASO requires a specific inversion time to null the blood signal and thus lengthens the sampling time of the combined sequence (Huber et al., 2017).

Ultimately, the selection of acquisition schemes and specific imaging parameters cannot be undertaken blindly. Each decision can directly influence the accuracy and precision of the resulting physiological maps. In the next section, we will continue to explore the assumptions of our physiological models, and how variance and uncertainty can be identified and addressed.

\section{$4.2 \alpha$}

As introduced in Section 2, the parameter $\alpha$ is a power-law coefficient describing the coupling between CBF and CBV. Grubb et al. (1974) assessed vascular responses to graded hypercapnia in anaesthetised rhesus monkeys using PET, observing a linear increase in total $\mathrm{CBV}$ with increasing $\mathrm{CO}_{2}$ partial pressure, and accompanying non-linear dependence of $\mathrm{CBF}$, where $\mathrm{CBV}=0.8 \mathrm{CBF}^{\alpha}$, and $\alpha=0.38$. However, given that the BOLD signal arises predominantly from the venous compartment, in the context of calibrated fMRI, the Grubb constant may not be accurate as it accounts for total CBV. Indeed, a significantly lower coupling coefficient between CBF and venous CBV has been demonstrated using MRI and moderate hypercapnia (Chen and Pike, 2009), leading to many recent calibrated fMRI studies now assuming this lower value of $\alpha \sim 0.23$. It should be noted that this value is specific to lowintensity sensorimotor stimulation, with lower and higher values of $\alpha$ reported for low and high intensity visual stimuli, respectively (Chen and Pike, 2009).

Detailed simulations have demonstrated that accurate determination of $\alpha$ is critical to the accuracy of estimates of the change in $\mathrm{CMRO}_{2}$ due to brain activity. An overestimation of $\alpha$ leads to an overestimation in calibration parameter $M$, and substantial underestimation in $\mathrm{CMRO}_{2}$, with greater error at larger changes in $\mathrm{CMRO}_{2}$ (Blockley et al., 2012; Griffeth and Buxton, 2011). By relaxing the physiological meaning of $\alpha$ and fitting it as a free parameter, Griffeth and Buxton (2011) found that $\alpha=0.14$ minimised error in $\mathrm{CMRO}_{2}$, which is in reasonable agreement with venous $\mathrm{CBV}$-based experimental measures. Given that plausible lower $\alpha$ values are reported for calibrated fMRI, it is critical in future experiments to consider the potential influence of the experimental protocol when selecting which value to use. For example, Mark \& Pike (2012) highlight the variability in $\alpha$ across hypercapnia and hyperoxia for both visual and motor tasks, and potential regional differences, particularly between cortical and subcortical structures, may need to be examined (Ances et al., 2008). 
Whilst currently assumed values of $\alpha$ are based on normal physiological conditions, uncoupling of CBF and CBV have been observed in a range of diseases (Carrera et al., 2011; Ellika et al., 2007; Lacalle-Aurioles et al., 2014; Talati et al., 2015) as well as under anaesthesia (Kolbitsch et al., 2001), and in response to psychoactive stimulants (Luo et al., 2009). Ideally, such uncoupling would be reflected by assuming an adjusted $\alpha$ in these populations, however, this is complicated by heterogeneity across the brain, and remains an area for future investigation.

\section{$4.3 \beta$}

The paramagnetic nature of $\mathrm{dHb}$ means that higher concentrations lead to an increase in the transverse relaxation rate $\left(R_{2}{ }^{*}=1 / T_{2}{ }^{*}\right)$. This relationship between susceptibility shift and change in $\mathrm{R}_{2}{ }^{*}$ is characterised by the parameter $\beta$, which ultimately reflects the microvascular anatomy. Deriving this value requires detailed understanding of how protons in the extravascular space diffuse through the magnetic gradients that surround vessels, specifically whether they fall within static or diffusive dephasing regimes. Based on initial analytical models and MonteCarlo simulations, $\beta=1.5$ was adopted for $1.5 \mathrm{~T}$, with estimates bound between 1.0 and 2.0 for small and large vessels respectively (Boxerman et al., 1995; He and Yablonskiy, 2007; Ogawa et al., 1993; Yablonskiy and Haacke, 1994). The vessel size limit, where the transition between dephasing regimes occurs, reduces as $B_{0}$ increases (Kennan et al., 1994), such that $\beta$ reduces at higher field strengths (typically $\beta=1.3$ for 3 T and $\beta=1.0$ for $7 \mathrm{~T}$ ).

Potential challenges with adopting these 'standard' values of $\beta$ are that early modelling approaches did not fully account for intravascular signal, and later experimental validations (Driver et al., 2010; Murphy et al., 2013b; Rossi et al., 2012; van der Zwaag et al., 2009) did not account for changes in intravascular signal induced by experimental manipulation of CBV (Croal et al., 2017a). Furthermore, there is a dependency of $\beta$ on echo time, a potential source of error that will vary with experimental protocol. There has been a recent shift towards linearising calibrated BOLD models (Blockley et al., 2015b; Griffeth et al., 2013), a simplification that has been shown to minimise error in OEF (Merola et al., 2016). By measuring $\Delta R_{2}{ }^{*}$ in response to a graded isocapnic hyperoxia challenge, Croal et al. (2017) demonstrated experimentally that such an assumption may be appropriate across all field strengths, as a nonlinear regime can be sufficiently described as linear across the limited range of $[\mathrm{dHb}]$ seen in calibrated $\mathrm{fMRI}$ experiments. It is also plausible that $\beta$ might also vary under conditions such as disease, if pathology were to disrupt microvascular/macrovascular 
distributions (such as may be seen in the chaotic vasculature in some tumours). However, this remains a rather unexplored area.

\subsection{BOLD scaling parameter, $M$}

The BOLD scaling parameter, $M$, is one of the characteristic features of calibrated fMRI. Theoretically, $M$ represents the maximum BOLD signal change that would occur if all of the deoxyhaemoglobin could be washed out of the venous blood vessels. Measuring $M$ directly in this way would only be possible if CBF was sufficiently high or if oxygen metabolism could be suspended, which is implausible in practice. Therefore, calibration experiments generally only produce minor physiological perturbations that allow $M$ to be estimated with the Davis model. As noted earlier, this most commonly consists of either a hypercapnic or hyperoxic gas challenge. Table 1 provides an overview of the expected effects of gas challenges on both physiological parameters and model constants in healthy volunteers when inspiring altered levels of $\mathrm{CO}_{2}$ or $\mathrm{O}_{2}$ at levels typically used in calibrated fMRI experiments.

Hypercapnia challenges result in an increase in CBF and a decrease in venous [dHb]. Under the assumption that hypercapnia does not alter baseline $\mathrm{CMRO}_{2}$, the Davis model can be rewritten in terms of changes in the BOLD signal and CBF (Hoge et al., 1999).

$$
\frac{\triangle B O L D}{B O L D_{0}}=M\left(1-\left(\frac{C B F}{C B F_{0}}\right)^{\alpha-\beta}\right)
$$

By rearranging Eq. 4 and combining the acquired BOLD and CBF measurements from a hypercapnia challenge, $M$ can be estimated. As $M$ is the change in $\mathrm{T}_{2}{ }^{*}$ weighted signal that would be caused by a total washout of $\mathrm{dHb}$ from the voxel, its amplitude is determined by properties of the tissue, the pulse sequence, and the amount of $\mathrm{dHb}$ present at rest:

$$
M=T E A C B V_{0}[d H b]_{0}^{\beta},
$$

where TE is the pulse sequence echo time and $A$ is a field strength and sample-specific proportionality constant (Hoge et al., 1999).

The discomfort experienced by some participants during hypercapnia prompted the development of the hyperoxia calibration technique. In contrast to hypercapnia, hyperoxia is imperceptible to participants and reduces $\mathrm{dHb}$ content by increasing the amount of $\mathrm{O}_{2}$ carried in the arterial blood plasma. In turn, more $\mathrm{O}_{2}$ remains bound to haemoglobin in the venous 
blood vessels, as the plasma $\mathrm{O}_{2}$ is preferentially consumed, resulting in a reduced $[\mathrm{dHb}]$. Again, the Davis model can be rearranged to describe this experiment:

$$
\frac{\triangle B O L D}{B O L D_{0}}=M\left(1-\left(1+\frac{\Delta[d H b]}{[d H b]_{0}}\right)^{\beta}\right)
$$

leaving us with an unknown change in venous $[\mathrm{dHb}](\Delta[\mathrm{dHb}])$. Fortunately, this can be estimated from measurements of the participant's $\mathrm{P}_{\mathrm{ET}} \mathrm{O}_{2}$, assuming equivalence with $\mathrm{PaO}_{2}$, using a gas analyser. Given this information it is possible to estimate the change in deoxyhaemoglobin concentration, $\Delta[\mathrm{dHb}]$, (Blockley et al., 2015b; Chiarelli et al., 2007),

$$
\Delta[d H b]=-\frac{\varphi[H b] \Delta S^{2} O_{2}+\varepsilon \Delta P a O_{2}}{\varphi}
$$

where $\mathrm{SaO}_{2}$ is the arterial blood oxygen saturation (Severinghaus, 1979), [Hb] is the haemoglobin concentration $([\mathrm{Hb}] \approx 0.03 \times \mathrm{Hct}), \phi$ is the oxygen carrying capacity of haemoglobin and $\varepsilon$ is the solubility coefficient of oxygen in blood. However, the resting deoxyhaemoglobin, $[\mathrm{dHb}]_{0}$, is unknown as it relies on a prior knowledge of the OEF (assuming $\mathrm{SaO}_{2}=1$ ):

$$
[d H b]_{0}=[H b] O E F,
$$

Therefore, in order to estimate $M$ in a hyperoxia calibration experiment using Eqs. 6 and 7, a value of OEF must be assumed. For a healthy population of participants, it may be reasonable to assume a single value of OEF. However, when comparing with an unhealthy cohort or an inherently heterogeneous group this may be a poor assumption as the hyperoxia calibration method does not cope well with such variance. Similarly, incorrect assumptions regarding Hct and $\mathrm{SaO}_{2}$ can negatively impact calibration via hyperoxia or combined hypercapnia-hyperoxia experiments. 


\begin{tabular}{|c|c|c|}
\hline Parameter & Hypercapnia & Hyperoxia \\
\hline CBF & $\begin{array}{l}\text { Increase } \\
\text { (Ito et al., 2008) }\end{array}$ & $\begin{array}{l}\text { Decrease/No change }^{\dagger} \\
\text { (Floyd et al., 2003, Croal et al., 2015) }\end{array}$ \\
\hline CBV & $\begin{array}{l}\text { Increase (arteries only) } \\
\text { (Ito et al., 2005) }\end{array}$ & $\begin{array}{l}\text { Decrease/No change }^{\dagger} \text { (arteries only) } \\
\text { (Kolbitsch et al., 2002, Croal et al., 2015) }\end{array}$ \\
\hline OEF & $\begin{array}{l}\text { Decrease } \\
\text { (Lin et al., 2013) }\end{array}$ & $\begin{array}{l}\text { Decrease } \\
\text { (Faraco et al., 2015b) }\end{array}$ \\
\hline $\mathrm{CMRO}_{2}$ & $\begin{array}{l}\text { Decrease/No Change } \\
\text { (See Table 2) }\end{array}$ & $\begin{array}{l}\text { Decrease/No change } \\
\text { (Sicard and Duong, 2005) }\end{array}$ \\
\hline$\alpha$ & $\begin{array}{l}\text { Decrease } \\
\text { (J. Jean Chen and Pike, 2010) }\end{array}$ & $\begin{array}{l}\text { No Change } \\
\text { (Faraco et al., 2015b) }\end{array}$ \\
\hline$\beta$ & No Change* & No Change* \\
\hline BOLD Signal & $\begin{array}{l}\text { Increase } \\
\text { (Mandell et al., 2008) }\end{array}$ & $\begin{array}{l}\text { Increase } \\
\text { (Losert et al., 2002) }\end{array}$ \\
\hline ASL: Perfusion & $\begin{array}{l}\text { Increase } \\
\text { (Noth et al., 2006) }\end{array}$ & $\begin{array}{l}\text { Decrease/No Change }{ }^{\dagger} \\
\text { (Bulte et al., 2007a) }\end{array}$ \\
\hline Blood $T_{2}$ & $\begin{array}{l}\text { Increase (veins only) } \\
\text { (Kettunen et al., 2002) }\end{array}$ & $\begin{array}{l}\text { No change } \\
\text { (Kettunen et al., 2002) }\end{array}$ \\
\hline Blood $T_{1}$ & $\begin{array}{l}\text { No change } \\
\text { (Faraco et al., 2015b) }\end{array}$ & $\begin{array}{l}\text { Decrease (arteries only) } \\
\text { (Kettunen et al., 2002) }\end{array}$ \\
\hline Blood $T_{2}{ }^{*}$ & $\begin{array}{l}\text { Increase (veins only) } \\
\text { (Faraco et al., 2015b) }\end{array}$ & $\begin{array}{l}\text { Increase (veins only) } \\
\text { (Faraco et al., 2015b) }\end{array}$ \\
\hline [dHb] & $\begin{array}{l}\text { Decrease } \\
\text { (Hoge et al., 1999) }\end{array}$ & $\begin{array}{l}\text { Decrease } \\
\text { (Driver et al., 2014) }\end{array}$ \\
\hline $\mathrm{SaO}_{2}$ & $\begin{array}{l}\text { No change } \\
\text { (Faraco et al., 2015b) }\end{array}$ & $\begin{array}{l}\text { Increase (slight) } \\
\text { (Faraco et al., 2015b) }\end{array}$ \\
\hline $\mathrm{SvO}_{2}$ & $\begin{array}{l}\text { Increase } \\
\text { (Faraco et al., 2015b) }\end{array}$ & $\begin{array}{l}\text { Increase } \\
\text { (Faraco et al., 2015b) }\end{array}$ \\
\hline
\end{tabular}


Table 1. Indication of the impact on physiological and model parameters in healthy subjects of altering the inspired fractions of carbon dioxide or oxygen to levels commonly used for calibration. Hypercapnia and hyperoxia refer to $\mathrm{FiCO}_{2} \sim 0.05$ and $\mathrm{FiO}_{2} \sim 0.5$, respectively.

Hct typically ranges between 36-54\% (Billett, 1990), with natural fluctuations arising as a function of age, (Adeli et al., 2015; Daniel, 1973) and potentially race (Beutler and West, 2017; Cheng et al., 2004). The need for individualised knowledge of Hct becomes critical in anaemia, with significant reductions observed in a wide range of pathology (Babitt and Lin, 2012; Goodnough et al., 2003; Muncie and Campbell, 2009; Rees et al., 2010; van Iperen et al., 2001), secondary to therapy (Groopman and Itri, 1999; Ludwig et al., 2004; Rodgers et al., 2012), and in response to poor nutrition (Baker and Demaeyer, 1979). Both invasive and noninvasive methods exist for Hct quantification, with the typical clinical approach being a packedcell volume test as part of a complete blood count (thus requiring a blood sample). Whilst this is the most efficient and accurate, there are circumstances where this may not be desirable from either a medical (e.g. neonatal subjects) or ethical perspective (e.g. healthy control studies). Potential alternatives include a multi-wavelength spectrophotometric method called co-oximetry, or an MR-derived measure that utilises the linear dependence of $\mathrm{T}_{1}$ on Hct, however both remain susceptible to error (Joseph et al., 2016; Li et al., 2017). Furthermore, Hct is known to be reduced in microvasculature in comparison to larger vessels. This can be addressed by assuming a lower uniform Hct value of $\sim 0.25$ (Calamante et al., 2016), however regional variation in Hct (Okazawa et al., 1996) may still contribute variance, and pathologies such as haemodialysis and ischaemia may further disrupt vascular anatomy (Mchedlishvili and Varazashvili, 1987; Mitra et al., 2004).

Arterial oxygen saturation $\left(\mathrm{SaO}_{2}\right)$ represents the percentage of haemoglobin molecules saturated with oxygen in the arteries, which is typically $95-100 \%$ for healthy subjects under normal conditions. Assuming fully oxygenated arterial blood $\left(\mathrm{SaO}_{2}=100 \%\right)$ is appealing and commonly done, as it simplifies much of the modeling and estimation of $\Delta[\mathrm{dHb}]$, OEF and $\mathrm{CMRO}_{2}$. While Blockley et al. (Blockley et al., 2015b) demonstrated that for the generalised calibration model (Gauthier and Hoge, 2012b) this simplification could lead to potential errors across all three parameters, in practice, this error is negligible across normoxic ranges. However under hypoxic conditions, this would lead to potential underestimation of $\Delta[\mathrm{dHb}]$, and overestimation of $\mathrm{OEF}$ and $\mathrm{CMRO}_{2}$ (Blockley et al., 2015b). In clinical cohorts where significant reductions in $\mathrm{SaO}_{2}$ (desaturation) are expected, it may be important to empirically 
measure this value. While the gold-standard technique is multi-wavelength co-oximetry and blood sampling, $\mathrm{SaO}_{2}$ is most commonly estimated using a pulse oximeter, which offers a noninvasive (and often MR-compatible) transcutaneous approach but remains influenced by probe positioning and limb temperature (Schramm et al., 1997). $\mathrm{SaO}_{2}$ can also be inferred from $\mathrm{PaO}_{2}$ via the modified Hill's equation (Severinghaus, 1979), and $\mathrm{PaO}_{2}$ can in turn be estimated from end-tidal $\mathrm{O}_{2}$ measurements throughout scanning.

Assuming that this variability can be accounted for, is $M$ simply a scaling factor in our calibration or is it ultimately useful in understanding an individual's physiology? It is sensitive to multiple aspects of physiology, including resting venous CBV, [dHb], and vessel size through $\beta$. It is also sensitive to multiple aspects of the MRI acquisition, echo time, magnetic field strength, and the difference in magnetic susceptibility between oxygenated and deoxygenated haemoglobin (Hare et al., 2015). This has led to a wide range of reported $M$ values, in addition to high test-retest variability (Lajoie et al., 2016). Therefore, we consider $M$ to be a useful model parameter and critical to calibration, but not a derived image that can be interpreted in our multiparametric physiological experiment.

\subsection{Effect of gas challenges}

A crucial assumption of the calibrated fMRI model is that the gas challenges we administer to induce BOLD, CBF, and CBV changes are isometabolic, meaning they will not influence the $\mathrm{CMRO}_{2}$ values we wish to measure. However, there is evidence that hypercapnia challenges can alter metabolism and brain activity patterns (Hall et al., 2011; Xu et al., 2011), and even small resting fluctuations in $\mathrm{CO}_{2}$ levels are associated with neural changes (Driver et al., 2016). Ultimately, there remains debate over whether the levels of hypercapnia typically achieved in calibrated fMRI experiments have a meaningful effect on $\mathrm{CMRO}_{2}$ estimates, and whether this can be corrected for (Bulte et al., 2012). A summary of the relevant literature is provided in Table 2. 
Kety-Schmidt Technique

(Kety and Schmidt, 1948)

PET

(Rhodes et al., 1981)

Radioactive Microspheres (Hoffman et al., 1982)

Radioactive Microspheres (Barzilay et al., 1985)

Kety-Schmidt Technique (Horvath et al., 1994)

Optical Imaging, LDF (Jones et al., 2005)

EEG

(Jones et al., 2005)

Calibrated fMRI

(Sicard and Duong, 2005)

Local Field Potentials

(Zappe et al., 2008)

Kety-Schmidt Technique (Chen and Pike, 2010)

\section{TRUST MR}

(Jain et al., 2011)

\section{TRUST MR}

(Xu et al., 2011)

\section{EEG}

(Xu et al., 2011)

MEG

(Hall et al., 2011)

MEG

(Thesen et al., 2012)

DOT, Calibrated fMRI

(Yucel et al., 2014)

ITRUST MRI

(Rodgers et al., 2015)

MEG

(Driver et al., 2016)

Calibrated fMRI

(Driver et al., 2017)

TRUST MRI

(Peng et al., 2017)
Humans

Dogs

Sprague-Dawley No change

Rats

Dogs

Male Fisher

Rats

Female Hooded Mixed

Lister Rats

Female Hooded Mixed

Lister Rats

Male Sprague- Mixed

Dawley Rats

Macaques

Humans

Humans

Humans

Humans

Humans

Humans

Humans

Humans

Humans

Humans

Humans

No change

No change

Mixed

Decrease

Decrease
No significant change with $5 \%$ or $7 \% \mathrm{CO}_{2}$ inhalation

No significant change with $+20-100 \mathrm{mmHg} \triangle \mathrm{P}_{\mathrm{ET}} \mathrm{CO}_{2}$

No significant change with $+20 \mathrm{mmHg} \triangle \mathrm{P}_{\mathrm{ET}} \mathrm{CO}_{2}$

No significant change with $+35 \mathrm{mmHg} \triangle \mathrm{P}_{\mathrm{ET}} \mathrm{CO}_{2}$

$60 \%$ increase with severe $\left(+22 \mathrm{mmHg} \Delta \mathrm{P}_{\mathrm{ET}} \mathrm{CO}_{2}\right)$ hypercapnia

No significant change with $5 \% \mathrm{CO}_{2}$ inhalation, significant increase $(\sim 30 \%)$ with $10 \% \mathrm{CO}_{2}$ inhalation

No significant change with $5 \% \mathrm{CO}_{2}$ inhalation, significant cortical desynchronisation with $10 \% \mathrm{CO}_{2}$ inhalation

No significant change with $5 \% \mathrm{CO}_{2}$ inhalation, significant decrease with $10 \% \mathrm{CO}_{2}$ inhalation

$15 \%$ decrease in beta and gamma frequency bands with $6 \% \mathrm{CO}_{2}$ inhalation

No significant change with mild $\left(+4.7 \mathrm{mmHg} \triangle \mathrm{P}_{\mathrm{ET}} \mathrm{CO}_{2}\right)$ or moderate $\left(+9.0 \mathrm{mmHg} \Delta \mathrm{P}_{\mathrm{ET}} \mathrm{CO}_{2}\right)$ hypercapnia

No significant change with $5 \% \mathrm{CO}_{2}$ inhalation

$13.4 \% \pm 2.3 \%$ decrease with inhalation of $5 \% \mathrm{CO}_{2}$; linear relationship between $\triangle \mathrm{CMRO}_{2}$ and $\triangle \mathrm{P}_{\mathrm{ET}} \mathrm{CO}_{2}$

Power significantly increased in delta band, decreased in alpha band with $5 \% \mathrm{CO}_{2}$ inhalation

Spatially-varying reduction in oscillatory power in alpha, beta, low gamma bands with $+6.7 \pm 0.7 \mathrm{mmHg} \triangle \mathrm{P}_{\mathrm{ET}} \mathrm{mO}_{2}$

Significant decrease in power in gamma band with $5 \%$ $\mathrm{CO}_{2}$ inhalation

No Change

Significant correlation between $\mathrm{M}$ derived from these two techniques with $+8 \mathrm{mmHg} \Delta \mathrm{P}_{\mathrm{ET}} \mathrm{CO}_{2}$

Significant $14.6 \% \pm 3.6 \%$ reduction using TRUST; no change using susceptometry, with $5 \% \mathrm{CO}_{2}$ inhalation

Decrease in oscillatory power in delta, alpha, beta, low gamma bands with $+0-8 \mathrm{mmHg} \triangle \mathrm{P}_{\mathrm{ET}} \mathrm{CO}_{2}$

Significant decreases of $1.5 \pm 0.6 \% / \mathrm{mmHg}$ (visual), $1.8 \pm$ $0.7 \% / \mathrm{mmHg}$ (motor), $1.3 \pm 0.4 \% / \mathrm{mmHg}$ (other $\mathrm{GM}$ )

Decrease in oscillatory power in delta, alpha, beta, low gamma bands with $5 \% \mathrm{CO}_{2}$ inhalation 
Table 2: Literature demonstrating the variable effects of hypercapnia on $\mathrm{CMRO}_{2}$ as measured using both MRI and non-MRI techniques.

The advent of the use of a hyperoxic stimulus for calibration introduced additional concerns about the potential impact of elevated $\mathrm{O}_{2}$ on metabolism and physiology. Using magnetoencephalography, Croal et al. (2015) observed a significant alteration in neuronal oscillations during hyperoxia at rest, albeit it of lower magnitude and more focal than hypercapnia. Studies using electroencephalography (EEG) have found a range of conflicting results, both at rest (Kaskinoro et al., 2010; Seo et al., 2007; Sheng et al., 2017a) and under activation (Sheng et al., 2017a; Smith and Strawbridge, 1974), which may in part reflect the limited spatial resolution of EEG.

There are additional physiological confounds which may need to be accounted for in hyperoxia calibrated MRI, depending on stimulus type. A small reduction in CBF has been reported using a fixed inspired challenge, due to vasoconstriction caused by both increased $\mathrm{PaO}_{2}$ and concurrent mild hyperventilation (inducing hypocapnia) (Bulte et al., 2007). By maintaining a constant $\mathrm{CO}_{2}$ level during the hyperoxia challenge, it was found that the flow velocity in the internal carotid arteries was not altered, suggesting that hypocapnia may be the dominant cause of this vasoconstrictive effect (Croal et al., 2015). Adding a further complication, changes in $\mathrm{PaO}_{2}$ have a direct influence on the $\mathrm{T}_{1}$ of blood, impacting the decay of the tagged blood signal (Silvennoinen et al., 2003; Tadamura et al., 1997). As the oxygen content of the blood plasma increases, $T_{1}$ decreases resulting in overestimation of the absolute change in CBF (Bulte et al., 2007). Note that these issues become even more complex if using carbogen (a mixture of $\mathrm{CO}_{2}$ and $\mathrm{O}_{2}$, with $\mathrm{CO}_{2}$ fractions typically ranging from 2 to $10 \%$ ), or any combination of altered $\mathrm{CO}_{2}$ and $\mathrm{O}_{2}$ (Hare et al., 2013), but can be minimised with isocapnic hyperoxia (Croal et al., 2015).

Initially, the SNR of ASL sequences was barely capable of detecting this small negative flow change. Thus, when using the model, it was usual to assume a degree of reduced flow in CBF rather than to rely on the ASL measured values. With advances in ASL acquisition, it is becoming possible to quantify small CBF changes and even AAT changes during hyperoxia calibration. $T_{1}$ effects can potentially be estimated using known relationships between arterial oxygenation and relaxation times from the literature (Zhao et al., 2007). Ultimately, when selecting a strategy for quantifying CBF for calibrated $\mathrm{FMRI}$, it is important to understand the assumptions of that approach and whether the gas challenges will require additional correction steps. 
Finally, the calibrated fMRI experiment also benefits greatly from long, steady-state periods of gas challenges. Unavoidable transients or resting fluctuations in arterial gas levels in addition to the intended gas stimulus paradigm will add noise to our acquired fMRI data, and ways to characterise and minimise these effects will boost the sensitivity and accuracy of calibration. There is substantial ongoing effort to induce stable changes in arterial gas tensions, independent of changes in the participant's ventilatory patterns (Slessarev et al., 2007; Wise et al., 2007).

\section{Derived parameters}

In the analysis of calibrated fMRI data, the acquired images described in Section 3 are combined using the Davis Model (Section 2), with careful attention paid to the model parameters and assumptions described in Section 4. The result is maps of new physiological parameters that cannot be measured directly, but that have clear clinical relevance in understanding brain health.

\subsection{OEF}

The OEF is simply the fraction of haemoglobin-bound oxygen that is extracted from the blood during its transit through the capillaries. It is defined as:

$$
O E F=\left(\frac{S_{a} O_{2}-S_{v} O_{2}}{S_{a} O_{2}}\right),
$$

where $\mathrm{SaO}_{2}$ is the arterial blood oxygen saturation (incoming blood oxygenation) and $\mathrm{SvO}_{2}$ is the venous blood oxygen saturation (outgoing blood oxygenation). As already discussed, $\mathrm{SaO}_{2}$ is often assumed to be 1 in healthy subjects with $\mathrm{FiO}_{2}>0.2$, but can be measured for greater accuracy.

Whole-brain measures of OEF can be obtained with MRI (Lu and Ge, 2008). However, obtaining regional maps is more challenging. As described in Section 2, conventional calibrated fMRI experiments using a single hypercapnic or hyperoxic calibration do not contain sufficient information to estimate OEF. However, dual calibrated fMRI methods, which involve administering both $\mathrm{O}_{2}$ and $\mathrm{CO}_{2}$ gas challenges, enable measurement of baseline OEF that can then be combined with CBF maps to achieve quantitative maps of resting $\mathrm{CMRO}_{2}$ (see section 5.2). Three major variants of dual calibrated fMRI exist, however, with differences in 
the implementation of the model fitting and parameter estimation leading to key differences in error and noise propagation.

The simplest approach involves the serial calculation of each model parameter (Bulte et al., 2012), resulting in a weighting of error towards the last parameters calculated, i.e. baseline $\mathrm{OEF}$ and baseline $\mathrm{CMRO}_{2}$. This approach means that images from the early stages of calculations have the highest SNR, but those at the end of the process (and arguably the most interesting) have the lowest. Another issue with this approach is that decisions about the analysis have to be made at each step. For example, should any of the data be smoothed, and if so, when and by how much? Data obtained from this method are shown in Figure 2. Other groups took a more simultaneous approach to the calculations (Gauthier and Hoge, 2012b; Wise et al., 2013): the total amount of noise in the data is the same, but it is distributed more evenly, resulting in a higher SNR in the derived baseline OEF (or $\mathrm{CMRO}_{2}$ ) image.

Whilst a detailed description of these data analysis approaches is beyond the scope of this review, here, an illustration of the basic principles is presented. A greater insight can be gained by considering a simplification of the approach proposed by Bulte et al. (2012). Under the assumption $\beta=1$, Eq. (4), describing the hypercapnia $(\mathrm{HC})$ calibration experiment, can be rewritten in the following way, substituting Eq. (5) for M:

$$
\frac{\triangle B O L D^{H C}}{B O L D_{0}}=\underbrace{T E A C B V_{0}[d H b]_{0}}_{M}\left(1-\left(\frac{C B F^{H C}}{C B F_{0}}\right)^{\alpha-1}\right),
$$

Given measurements of the change in BOLD and CBF, we can produce a signal that is sensitive to the product of $\mathrm{CBV}_{0}$ and $[\mathrm{dHb}]_{0}$ (plus some constants). Using a similar approach Eq. (6) describing the hyperoxia $(\mathrm{HO})$ calibration experiment can be rewritten as

$$
\frac{\triangle B O L D^{H O}}{B O L D_{0}}=-T E A C B V_{0} \Delta[d H b]
$$

At first glance this looks significantly different to Eq. (6). However, it demonstrates the insensitivity of the hyperoxia experiment to $[\mathrm{dHb}]_{0}$ (and hence OEF via Eq. (8)). This also explains the need to assume a value for OEF in conventional calibrated fMRI using hyperoxia. Importantly, Eq. (11) demonstrates that the BOLD signal change in response to hyperoxia is $\mathrm{CBV}_{0}$ weighted. Given a BOLD measurement and an estimate of $\Delta[\mathrm{dHb}]$ (see Eq. (7)), the signal can be sensitised to $\mathrm{CBV}_{0}$ (plus some constants). Finally, by taking the ratio of Eq. (10) 
and (11), and rearranging for $[\mathrm{dHb}]_{0}$, it can be seen that these constants do not need to be known (Blockley et al., 2015b):

$$
[d H b]_{0}=\frac{\left(\frac{\Delta B O L D^{H C}}{B O L D_{0}}\right)}{\left(\frac{\Delta B O L D^{H O}}{B O L D_{0}}\right)} \frac{\Delta[d H b]_{0}}{\left(1-\left(\frac{C B F H C}{C B F_{0}}\right)^{\alpha-1}\right)} .
$$

This simplified dual calibrated fMRI model highlights the roles that hypercapnia and hyperoxia challenges play. The hypercapnia BOLD signal is sensitive to both CBV and [dHb], whilst the hyperoxia BOLD signal is sensitive to CBV alone, enabling $[\mathrm{dHb}]$ to be extracted and OEF calculated using Eq. (8).

The dual calibrated fMRI approach, despite variation in the order of calculations, results in quantitative images of baseline OEF using data collected with whole brain coverage at typical BOLD resolutions in under 20 minutes. Although more complex than single gas calibrated fMRI in terms of both experiment design and modeling, knowledge of baseline OEF (and $\mathrm{CMRO}_{2}$ ) has greater clinical potential, for both understanding pathophysiological alterations and treatment monitoring (De Vis et al., 2015; Lajoie et al., 2017).

\section{$5.2 \mathrm{CMRO}_{2}$}

Oxidative metabolism is necessary to meet the vast majority of the brain's energetic demands, and therefore believed to be the physiological parameter most tightly coupled to the activity of the brain (Du et al., 2008; Hyder, 2010). Hence $\mathrm{CMRO}_{2}$ is an important biomarker for cerebrovascular health, providing the best measure of either resting activity or relative changes during periods of increased activity.

Relative changes in $\mathrm{CMRO}_{2}$ can be obtained from a conventional calibrated fMRI scan based on the Davis model (Eq. 2). Although much of the research on relative $\mathrm{CMRO}_{2}$ has focused on validation within healthy populations, there is demonstrated utility under disrupted physiology such as in ageing (Mohtasib et al., 2012), multiple sclerosis (Hubbard et al., 2017) and HIV (Ances et al., 2011).

In addition to characterising relative changes in metabolism, quantitative measures of tissue metabolism at rest may provide an important clinical biomarker. Resting $\mathrm{CMRO}_{2}$ at baseline is essentially defined from Fick's principle that the oxygen delivered to a region minus the oxygen departing that region must be the amount of oxygen left behind and consequently 
metabolised. Thus, to calculate resting $\mathrm{CMRO}_{2}$ we need to know resting $\mathrm{CBF}$ (the rate of blood delivery), resting OEF (the fraction of oxygen extracted for metabolism), and the oxygen carrying capacity of blood $\left(\mathrm{C}_{a} \mathrm{O}_{2}\right)$, which can be inferred from Hct.

$$
\mathrm{CMRO}_{2}=\mathrm{CBF} \text { OEF } \mathrm{C}_{a} \mathrm{O}_{2}
$$

Thus, resting $\mathrm{CMRO}_{2}$ images can be produced by multiplying and scaling quantitative images of resting OEF and CBF.
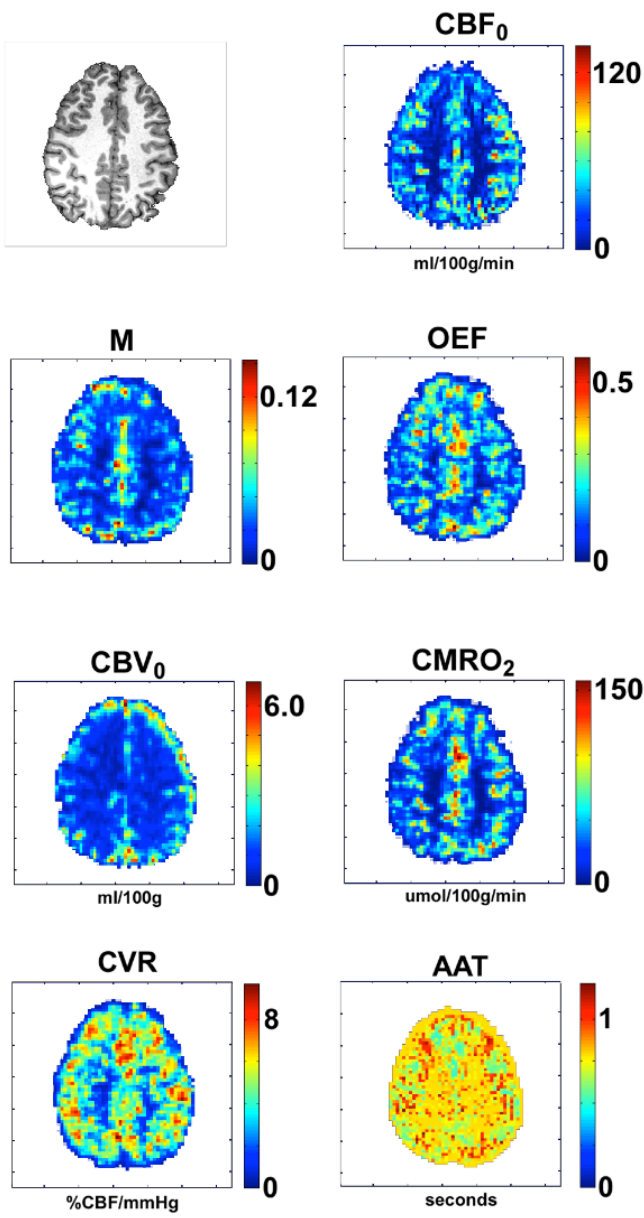

Figure 2. Single slice from an example dual calibrated fMRI dataset. Top left shows the high-resolution $\mathrm{T}_{1}$-weighted anatomical image. The CBF and AAT images were produced from a multi-PLD pCASL sequence, $M$ was calculated from a hypercapnia stimulus, OEF was determined from $M$ and the BOLD response to a hyperoxia stimulus. CBV was calculated from the $\mathrm{BOLD}$ response to hyperoxia. $\mathrm{CMRO}_{2}$ was calculated from CBF and OEF, and the CVR was calculated from the ASL response to hypercapnia. 


\subsection{Cerebrovascular Reactivity}

Monitoring $\mathrm{P}_{\mathrm{ET}} \mathrm{CO}_{2}$ during $\mathrm{BOLD}$ or $\mathrm{CBF}$ imaging enables robust mapping of cerebrovascular reactivity (CVR), the vascular response to a vasoactive stimulus. Routinely assessed in large vessels using TCD and simple breath-holding techniques that cause transient hypercapnia (Müller et al., 1995), CVR provides insight into healthy or pathological vascular function. When CVR is acquired in the context of a calibrated fMRI experiment, it provides semi-quantitative or quantitative maps of \%BOLD or \%CBF increase per $\triangle \mathrm{P}_{\mathrm{ET}} \mathrm{CO}_{2}(\mathrm{mmHg})$ throughout the brain.

CVR maps are sensitive to regional vascular pathology in a range of diseases. In a recent review article, Juttokanda and Donahue present an excellent overview of the clinical CVR literature in cerebrovascular disease, highlighting the role of CVR imaging in studying arterial steno-occlusive disease, anemia, and small vessel disease (Juttukonda et al., 2017). Special care needs to be taken when using ASL to quantify CVR in these patient cohorts, as many assumptions about the labelling efficiency or vascular transit times may be altered in the presence of widespread vascular pathology (as discussed in Section 3.2).

Dementia is another key target for CVR research. In young healthy carriers of ApoE-e4, a genetic risk factor for Alzheimer's Disease (AD), BOLD CVR has identified haemodynamic impairment in specific brain regions associated with memory (Suri et al., 2015). In older patients with $A D$, altered regional CVR has been observed to correlate with cognitive function as assessed by the Mini Mental State Examination (Richiardi et al., 2015).

In healthy aging, CVR changes may also precede radiological and cognitive symptoms of agerelated white matter disease. After observing that areas of reduced CVR in healthy young individuals corresponded to regions of high white matter lesion frequency in the elderly, Sam and colleagues tested this relationship longitudinally, demonstrating that reduced CVR preceded the formation of white matter hyperintensities (Sam et al., 2016). CVR is also reduced in Multiple Sclerosis (MS), and this vascular impairment is correlated with grey matter atrophy and lesion volume (Marshall et al., 2014). Although typically viewed as a neuroinflammatory disease, these observations may indicate that haemodynamic impairment contributes to concurrent widespread neurodegeneration in MS. CVR is an exciting technique for monitoring and potentially predicting local tissue damage, and evaluating haemodynamic function in a range of diseases and at-risk groups. 
There is an ongoing effort to standardize the acquisition and reporting of CVR maps, as the international community has reported using numerous gas delivery protocols and imaging parameters that may not be directly comparable (Moreton et al., 2016). However, CVR is poised to become a clinical tool, as safety concerns are addressed (Spano et al., 2013) and clear targets for influencing clinical outcomes are identified (Smeeing et al., 2016). The CVR field has developed bespoke gas stimuli to efficiently probe different aspects of the vascular dilatory response, such as sinusoidal (Blockley et al., 2017) or ramped (Bhogal et al., 2014) delivery of hypercapnia, and it remains to be seen whether these techniques will be incorporated into calibrated fMRI experiments.

\section{Utility of multiparametric physiologic imaging}

We have thus far examined the numerous physiological parameters that can be extracted in the context of a calibrated fMRI experiment, including BOLD, CBF, CBV, CVR, OEF, and $\mathrm{CMRO}_{2}$ (e.g., Figure 2), but what is ultimately the benefit of achieving such multiparametric data?

In a study of traumatic brain injury (TBI), multiparametric physiological imaging data acquired within 72 hours of injury using ${ }^{15} \mathrm{O}$ PET were used to predict irreparable tissue damage (Cunningham et al., 2005). Although healthy thresholds for each parameter could be estimated, the study showed that multivariate analysis combining $\mathrm{CBF}, \mathrm{OEF}$, and $\mathrm{CMRO}_{2}$ could better predict lesion formation. The authors state:

"It is important to emphasize that [...] none of the physiological variables alone can be used to define acutely the extent of irreversibly damaged tissue. [...] Future attempts to produce predictive maps for tissue outcome may require using combinations of the physiological variables."

In this paper, we have highlighted MRI techniques for achieving multiparametric physiological data in the context of a calibrated fMRI experiment; we propose that these data will become increasingly important for accurately and precisely characterising neurovascular health in a range of clinical applications.

The following examples demonstrate that combining information across vascular and metabolic imaging metrics may pave the way for improved diagnosis in diseases with similar clinical presentations, enable staging of disease severity based on imaging metrics, and allow better development and targeting of treatment therapies. 


\subsection{Stroke}

Stroke is perhaps the most immediate candidate for multiparametric physiological imaging, as numerous haemodynamic changes occur concurrently with neural pathology and cognitive symptoms.

In the early stages of haemodynamic failure, autoregulatory compensation enables successful maintenance of CBF during a decrease in perfusion pressure via increases in CBV. As the limit of vasodilation (CBV) is reached and CBF is slightly reduced, an increase in OEF maintains normal $\mathrm{CMRO}_{2}$ (Powers, 1991); this stage is referred to as oligaemia. Eventually $\mathrm{CBF}$ is no longer sufficient, OEF is maximised, and $\mathrm{CMRO}_{2}$ is affected as acute damage is done to ischaemic brain tissue. By measuring $\mathrm{CBF}, \mathrm{CBV}, \mathrm{OEF}$ and $\mathrm{CMRO}_{2}$ in a multiparametric experiment, we can potentially stage the haemodynamic risk or damage to regional brain tissue.

Numerous studies in the literature have used ${ }^{15} \mathrm{O}$ PET to examine how combinations of physiological parameters can be used to assess subsequent risk to regional brain tissue in stroke. In a study of unilateral carotid artery occlusion, patients with increased OEF and CBV had higher risk of ischaemic stroke than those with increased OEF and normal CBV, suggesting more severe haemodynamic compromise, perhaps linked to exhausted autoregulatory vasodilation (Derdeyn et al., 2002). Reduced CBF and normal CVR may characterize oxygen hypometabolism, potentially due to ischemia-related neuronal loss (Kuroda et al., 2004) which may indicate that such ischemic lesions are not critically compromised from a haemodynamic perspective and are at low risk for subsequent stroke. Observations of normal CBF and reduced CVR may also reflect good prognosis (Isozaki et al., 2010). A negative correlation is observed between CVR and OEF in patients with occluded carotid arteries, potentially reflecting a continuous transition of tissue from normal conditions to increasing ischemic stress and beyond (Nemoto et al., 2004). However, the authors note key discrepancies in this relationship, and suggest that "OEF alone may not be capable of identifying the true spectrum of patients at increased stroke risk." These results from PET literature clearly demonstrate the promise of multiparametric imaging approaches to understanding the complex haemodynamic changes in stroke, and the MRI methods presented in this paper could be used to make such measurements more accessible and repeatable in routine clinical practice. 
Multiparametric physiological imaging may also improve our ability to plan treatment in stroke. In a study in rats, resting CBF and CVR were initially elevated in the peri-lesional cortex at 7 days post-infarct by $60 \%$ and $80 \%$, respectively (Lake et al., 2017). However, by 21 days after stroke, this CBF effect was resolved whereas CVR remained elevated. By combining CBF and CVR mapping of the peri-infarct zone in the weeks following an ischaemic insult, the authors suggest an extended temporal window for therapeutic interventions may be identified. In humans, CVR mapping in stroke has allowed compensatory flow patterns and vascular steal to be studied (Faraco et al., 2015a; Poublanc et al., 2013; Sobczyk et al., 2014). Multiparametric information has also been used to aid surgical planning and to monitor the effects of interventions in stroke (Donahue et al., 2017).

After stroke, imaging can also be used to monitor recovery of both haemodynamic and cerebral function. While CBV and CBF measures can identify angiogenesis, CVR can assess whether the newly generated vasculature is functionally capable. Therapeutic interventions can be administered to induce angiogenesis in specific brain regions: for example, exercise has been shown to increase CBV in the motor cortex of rats. Furthermore, whereas baseline CBF was maintained, CVR during hypercapnia was elevated in these regions, indicating that the exercise therapy increased the size of the capillary reserve (Swain et al., 2003). Finally, combining baseline measures of $\mathrm{CMRO}_{2}$ with functional BOLD changes during a stimulus can identify where vascular plasticity is directly fostering neural plasticity (Seevinck et al., 2010). Multiparametric imaging can thus play an important role in assessing the success of therapeutic interventions.

\subsection{Multiple Sclerosis}

Calibrated fMRI experiments have already been used successfully to study Multiple Sclerosis (MS), demonstrating a clear link between functional $\mathrm{CMRO}_{2}$ changes and fatigue and neurological disability across patients (Hubbard et al., 2017). With this demonstration of calibrated $\mathrm{fMRI}$ as a practical and informative technique in this cohort, and with evidence from the literature that numerous haemodynamic factors may contribute to or be affected in MS, we assert that multiparametric physiological data will greatly improve our understanding of the vascular contributions to this primarily neuroinflammatory and neurodegenerative disease.

For example, imaging haemodynamic changes may help track lesion formation and evolution. In an animal model of MS, lesion formation and BBB disruption are preceded by elevated CBV in the nearby tissue (Broom et al., 2005). In humans, active (contrast-enhancing) lesions also 
demonstrate approximately $25 \%$ increases in $\mathrm{CBV}$ and $\mathrm{CBF}$, and these haemodynamic changes are detectable prior to BBB breakdown or changes in tissue integrity (Ge et al., 2005; Wuerfel et al., 2004). This inflammatory vasodilation subsides over time and ultimately reverses: chronic lesions that persist beyond the acute inflammatory period exhibit reductions in CBV and CBF. Finally, these haemodynamic changes extend far beyond the focal lesion, affecting substantial areas of nearby tissue (Bester et al., 2015).

Many of these studies primarily relied on DSC imaging to measure CBF and CBV in humans, however these techniques may not be appropriate for regular, repeated imaging of haemodynamics in longitudinal studies. The Gadolinium-based contrast agents used in DSC acquisitions have recently been observed to accumulate in the brain in a dose-dependent manner (McDonald et al., 2015), and thus the imaging techniques used in calibrated fMRI (i.e., BOLD, ASL, VASO), which do not involve injection of these contrast agents, may be preferable to study acute vascular changes during periods of active disease.

CVR, OEF, and $\mathrm{CMRO}_{2}$ (baseline) have also been shown to be reduced in MS across the brain (by approximately $30 \%, 14 \%, 23 \%$, respectively), and these metrics appear to be associated with lesion load and atrophy measures (Ge et al., 2012; Marshall et al., 2014). Further characterisation of this impairment could elucidate whether CVR impairment or hypometabolism have a causal role in grey matter thinning or widespread neurodegeneration.

\subsection{Alzheimer's Disease and Dementia}

Characterisation of multiple haemodynamic factors may assist diagnosing and tracking the progression of Alzheimer's Disease (AD). In a comparison of patients with $A D$, patients with mild cognitive impairment who would later convert to $A D$, and healthy controls, CBV changes were observed only in the late-stage $A D$ cohort whereas $C B F$ impairment was observed earlier in disease progression (Lacalle-Aurioles et al., 2014). Impaired CVR has also been observed in young healthy subjects with increased genetic risk of developing AD (Suri et al., 2015). Thus, CBF and CVR mapping may offer early windows for understanding disease mechanisms, before changes in CBV or overt cognitive function develop.

It is also important to differentiate AD from other types of dementia (e.g., vascular or frontoparietal dementias). Lajoie and colleagues observed patterns of reduced $\mathrm{CBF}$ and $\mathrm{CMRO}_{2}$ that were specific to parieto-temporal brain regions known to be impaired in AD (Lajoie et al., 2017). When combined with unimpaired frontal CBF and normal whole-brain grey matter CVR, 
the researchers could more confidently assert that the observed pathology was associated specifically with $A D$ rather than generalised vascular disease. Similarly, in a study directly comparing $A D$ with vascular dementia, the combination of CBF and CVR mapping was sensitive to differences in pathological haemodynamics between the cohorts (Gao et al., 2013).

\subsection{Drug effects}

It is essential that multiple haemodynamic and metabolic parameters are characterised in drug studies. For example, sildenafil is a candidate treatment for AD patients; using MRI, a $50 \mathrm{mg}$ dose was shown to increase $\mathrm{CBF}$ and $\mathrm{CMRO}_{2}$ throughout the brain, with most pronounced CBF increases in bilateral medial temporal cortex, whereas CVR was decreased (Sheng et al., 2017b). Anaesthestic drugs also have numerous physiological consequences: both baseline and task-induced changes in CBF are reduced following low-dose sevoflurane, and the relationship between functional BOLD and CBF changes is impacted in a spatially nonuniform manner (Qiu et al., 2008).

In a dual calibrated fMRI experiment, Merola and colleagues explored the effects of caffeine, a common drug that may influence and add variability to functional imaging datasets in many studies (Merola et al., 2017). Compared to placebo, caffeine increased OEF (15.6\%) and decreased $\mathrm{CBF}(-30.4 \%)$ and $\mathrm{CMRO}_{2}(-18.6 \%)$ in healthy volunteers. Whereas the CBF effect was widespread throughout the brain, the metabolic changes were more localised to the frontal gyrus, parietal and occipital lobes. This study demonstrates the ability of calibrated fMRI experiments to map the vascular and metabolic effects of caffeine, and provides evidence that caffeine use (acute and chronic) should be considered and controlled in imaging studies when possible.

More generally, functional imaging studies to understand brain function during anaesthesia or following administration of a drug or medication must consider how neurovascular coupling relationships are affected, and calibrated $\mathrm{fMRI}$ studies will better identify which physiological parameters are truly being modulated in these cases.

\section{Considerations and Opportunities}

\subsection{Validation}


All data acquired in a calibrated fMRI experiment can be dramatically altered by changes in the imaging parameters or analysis procedures. However, CBF, CBV, OEF, $\mathrm{CMRO}_{2}$, and CVR (in terms of flow changes) are ultimately quantifying true human physiology that has been studied for centuries using a broad range of techniques. We have a body of literature that can provide estimates of these values in healthy humans and in specific cohorts, and many aspects of calibrated fMRI have been independently validated against other imaging modalities in a limited number of patient groups as well as with healthy subjects. The validation of calibrated fMRI is considered elsewhere in this issue and so will only be briefly considered here (see article by Wise et al. in this issue).

The use of different variants of ASL to measure CBF have been validated against PET (Fan et al., 2016), perfusion CT (Koziak et al., 2008), and transcranial Doppler Ultrasound (TCD) (Croal et al., 2017b). The primary clinical tool for measuring CVR in patients is TCD, and the most common vasoactive stimulus used is a short breath hold, although acetazolamide is often used in clinical settings to provide a more dramatic dilation effect. Both ASL and BOLD MRI have been used to measure CVR in patients (Smeeing et al., 2016) and healthy subjects (Zhou et al., 2015), and have been shown to correlate well. CBV has typically been measured with MRI using contrast enhanced methods involving the injection of a gadolinium-based contrast agent, which have in turn been validated in patients against SPECT methods (Engvall et al., 2008). The non-invasive MR method VASO has been validated against PET (Uh et al., 2011). OEF and $\mathrm{CMRO}_{2}$ can be measured using triple oxygen $\left({ }^{15} \mathrm{O}\right) \mathrm{PET}$, however the recent MRI methods for obtaining these parameters are yet to be validated against this modality. OEF information can also be obtained from near infrared spectroscopy (NIRS), however this is a mixed arterial-venous signal and only provides information over large, diffuse regions and thus is difficult to use as a direct validation tool.

Ultimately, the development of new physiological imaging parameters must be done with caution. There is constant risk of "fixing" acquisition procedures or "optimising" analysis pipelines such that the final numbers we achieve are within our expected, plausible limits as determined by gold-standard techniques or ranges published in the literature. As a field, we must be constantly sceptical of our credible results, and continue to push for confidence in our data through improved validation procedures.

\subsection{Calibration without gas challenges}


A multiparametric physiological imaging study of AD patients and healthy controls demonstrated the challenges encountered when administering gas challenges for calibrated fMRI in patient cohorts (Lajoie et al., 2017). During hypercapnic testing, 10 out of 59 patients $(17 \%)$ and 6 out of 60 controls $(10 \%)$ withdrew due to the feeling of anxiety caused by the $\mathrm{CO}_{2}$ inhalation. Mask leakage affected a further 6 patients and 2 controls. Interestingly, the frequency of drop-out or other problems related to hypercapnia and mask leakage was attenuated as the study trial progressed, indicating that researchers became more skilled at fixing leaky face masks via repositioning or tape, and at identifying which participants were okay to continue with the gas protocol (potentially introducing bias). The authors also note that the lengthy testing and imaging protocol was performed during a single visit, and suggest that "future calibrated studies may consider planning for a separate day to perform the hypercapnia testing alone, thus reducing potential stress and fatigue experienced by the participants." However, this study demonstrates that many patients and healthy controls may not be able to complete a calibrated fMRI experiment, and thus it is desirable to develop alternative approaches for achieving similar physiological data without the use of gas inhalation challenges.

In hypercapnic, hyperoxic, and dual calibration experiments, the physiological stimulus (modified gas inhalation) is used to physically reduce the amount of deoxyhaemoglobin present in the voxel, aiming to extrapolate to the condition where all $\mathrm{dHb}$ has been washed out of the venous blood. An alternative approach under development posits that we don't actually need to wash out the deoxyhaemoglobin, and instead we only need to remove its effect on the signal we measure (Blockley et al., 2015). Since the BOLD effect is caused by the dephasing of the tissue signal due to the microscopic magnetic field gradients that surround deoxygenated blood vessels, a spin echo refocusing pulse should be able to recover some of this lost signal. This "lost signal" can be measured by the relaxometry parameter $\mathrm{R}_{2}$ ', the reversible transverse relaxation rate. $R_{2}$ ' has previously been used to normalise stimulus evoked changes in $\mathrm{R}_{2}{ }^{*}$ on the way to quantifying changes in $\mathrm{CMRO}_{2}$ (Fujita et al., 2006; Kida et al., 2000). More recently $R_{2}{ }^{\prime}$ was placed in the calibrated $f M R I$ framework by recognising that it is theoretically sensitive to the same physical and physiological components as $M$ (c.f. Eq. 5),

$$
R_{2}^{\prime}=A C B V_{0}[d H b]_{0}^{\beta} \Rightarrow M=T E R_{2}^{\prime}
$$


However, the effectiveness of spin echo refocusing is dependent on the scale of the magnetic field gradients (Boxerman et al., 1995). Therefore, simulations predict that the $M$ measured via $\mathrm{R}_{2}$ ' will be underestimated when compared with the hypercapnia $M$ (Blockley et al., 2012b). This hypothesis was later confirmed experimentally (Blockley et al., 2015a).

The $R_{2}$ ' weighted signal can also be used to estimate baseline OEF using the closely related "quantitative BOLD" technique (He and Yablonskiy, 2007). Whilst the calibrated fMRI technique operates in the monoexponential regime of the $\mathrm{R}_{2}{ }^{*}$ signal, quantitative BOLD takes advantage of the additional information that is stored in the quadratic exponential decay. This quadratic exponential decay is revealed at low values of the spin echo displacement time, $\tau$, which is responsible for the degree of $\mathrm{R}_{2}$ ' weighting included in the image. This short $\tau$ regime provides sensitivity to venous CBV, enabling OEF to be extracted from a measurement of $R_{2}{ }^{\prime}$ (c.f. Eq. 8 and under the assumption $\beta=1$ ):

$$
O E F=\frac{R_{2}^{\prime}}{A[H b] C B V_{0}} .
$$

This principle was proven early on (An and Lin, 2000; He and Yablonskiy, 2007) with recent work concentrating on reducing confounding effects (Stone and Blockley, 2017) and robust model fitting (Domsch et al., 2017).

\subsection{Future opportunities}

Even after advanced correction strategies, several noise sources will inevitably remain in the acquired data in a calibrated fMRI experiment (Murphy et al., 2013a). In some instances, we may be able to characterise a noise source, model its impact on our data, and reduce or remove these confounds. We may also uncover new ways to transform these sources of variance into additional insight into brain function. The pulsatility of vessels, typically considered a noise source, can be combined with CBV estimates to achieve maps of arterial compliance, which is a useful measure of arterial stiffness (Yan et al., 2016). Blood pressure will affect numerous aspects of vascular physiology, and new hardware developments for measuring beat-to-beat blood pressure during scanning may allow us to characterise the vascular response to blood pressure fluctuations (Baruch et al., 2011; Whittaker et al., 2016). Advances in this area could lead to whole-brain assessment of cerebral autoregulation, which is a critical clinical measurement in hypertension and stroke (Diedler et al., 2009; Jordan and Powers, 2012; Paulson et al., 1990). Similarly, recording fluctuations in vascular tone (via pulse wave velocity) or arousal (using the Galvanic Skin Response as a surrogate) during the 
calibrated fMRI experiment could lead to interesting new directions for physiological parameter mapping.

Other sources of physiological variance may remain stable during the course of a single MRI scan session, but could add variance to a calibrated fMRI study when comparing individuals or cohorts. In a systematic review of factors contributing to CBF values, Clement and colleagues identified 58 factors that should be considered and/or controlled for in multi-centre ASL studies (Clement et al., 2017), grouped into four categories: a) physiology, lifestyle, and health, b) blood components, c) mental state, personality, and cognition, and d) drugs. Some parameters may be straightforward to control in a given study; for example, the time of day that scanning occurs could be fixed, the effect of meals or caffeine could be avoided via fasting, and smokers could be excluded. However, if smokers are the cohort of interest, it is important to use the complete multiparametric imaging power of calibrated fMRI to differentiate the vascular, metabolic, and neurovascular coupling changes in this cohort.

For more subtle contributors of physiological variance, such as mood or stress levels, large datasets may be necessary to probe their relationship to the multiple physiological parameters being mapped. As open-access data sharing becomes more supported, we encourage imagers to record as much physiological information about their participants as possible, to help future studies capitalise on any untapped information in these rich datasets.

\section{Conclusion}

Calibrating BOLD fMRI may seem daunting due to the requirement of additional hardware, pulse sequences, and analysis tools, as well as the additional "expertise" necessary to ensure the process goes smoothly. However, the added value of the extra physiological information that may be obtained, and the specificity and quantitation that enables the comparison of data between sessions, subjects, groups or sites, can more than compensate for this. As these techniques improve and become more commonplace, they will also benefit from further validation against more familiar modalities, and greater availability and reliability of the tools needed to perform the calibration. As with all neuroimaging, it is unlikely that there will ever be a calibration method that will work for every set of circumstances, however a variety of multiparametric methods will enable a researcher or clinician to select the approach that provides the most accurate and valuable diagnostic data specific to their needs. 


\section{Acknowledgments}

The authors receive support from the Engineering and Physical Sciences Research Council [grant number EP/K025716/1], Cancer Research UK, and the University of Nottingham Anne McLaren Fellowship programme.

\section{References}

Adeli, K., Raizman, J.E., Chen, Y., Higgins, V., Nieuwesteeg, M., Abdelhaleem, M., Wong, S.L., Blais, D., 2015. Complex Biological Profile of Hematologic Markers across Pediatric, Adult , and Geriatric Ages : Establishment of Robust Pediatric and Adult Reference Intervals on the Basis of the Canadian Health Measures Survey METHODS : Clin. Chem. 1086, 1075-1086. doi:10.1373/clinchem.2015.240531

An, H., Lin, W., 2000. Quantitative measurements of cerebral blood oxygen saturation using magnetic resonance imaging. J. Cereb. Blood Flow Metab. 20, 1225-36. doi:10.1097/00004647-200008000-00008

Ances, B., Vaida, F., Ellis, R., Buxton, R., 2011. Test-retest stability of calibrated BOLD-fMRI in HIV- and HIV+ subjects. Neuroimage 54, 2156-62. doi:10.1016/j.neuroimage.2010.09.081

Ances, B.M., Leontiev, O., Perthen, J.E., Liang, C., Lansing, A.E., Buxton, R.B., 2008. Regional differences in the coupling of cerebral blood flow and oxygen metabolism changes in response to activation: implications for BOLD-fMRI. Neuroimage 39, 151021. doi:10.1016/j.neuroimage.2007.11.015

Aslan, S., Xu, F., Wang, P.L., Uh, J., Yezhuvath, U.S., van Osch, M., Lu, H., 2010. Estimation of labeling efficiency in pseudocontinuous arterial spin labeling. Magn. Reson. Med. 63, 765-771. doi:10.1002/mrm.22245

Babitt, J.L., Lin, H.Y., 2012. Mechanisms of Anemia in CKD. J. Am. Soc. Nephrol. 16311634. doi:10.1681/ASN.2011111078

Baker, S.J., Demaeyer, E.M., 1979. Nutritional anemia: its understanding and control with special reference to the work of the World Health Organization. Perspect. Nutr. 32, 368-417.

Bandettini, P.A., 2012. Functional MRI: A confluence of fortunate circumstances. Neuroimage 61, A3-A11. doi:10.1016/j.neuroimage.2012.01.130

Bandettini, P.A., Wong, E.C., 1997. A hypercapnia-based normalization method for improved spatial localization of human brain activation with fMRI. NMR Biomed. 10, 197-203. doi:10.1002/(SICI)1099-1492(199706/08)10:4/5<197::AID-NBM466>3.0.CO;2-S

Bandettini, P.A., Wong, E.C., Hinks, R.S., Tikofsky, R.S., Hyde, J.S., 1992. Time course EPI of human brain function during task activation. Magn. Reson. Med. 25, 390-7.

Baruch, M.C., Warburton, D.E., Bredin, S.S., Cote, A., Gerdt, D.W., Adkins, C.M., 2011. Pulse Decomposition Analysis of the digital arterial pulse during hemorrhage simulation. Nonlinear Biomed. Phys. 5, 1. doi:10.1186/1753-4631-5-1

Barzilay, Z., Britten, a G., Koehler, R.C., Dean, J.M., Traystman, R.J., 1985. Interaction of $\mathrm{CO} 2$ and ammonia on cerebral blood flow and $\mathrm{O} 2$ consumption in dogs. Am. J. Physiol. 248, H500-H507.

Belliveau, J.W., Kwong, K.K., Kennedy, D.N., Baker, J.R., Stern, C.E., Benson, R., Chesler, D.A., Weisskoff, R.M., Cohen, M.S., Tootell, R.B., Fox, P.T., Brady, T.J., Rosen, B.R., 1992. Magnetic resonance imaging mapping of brain function. Human visual cortex. Invest. Radiol. 27, S59-65.

Berry, E.S.K., Jezzard, P., Okell, T.W., 2015. An Optimized Encoding Scheme for Planning Vessel-Encoded Pseudocontinuous Arterial Spin Labeling. Magn. Reson. Med. 74, 1248-56. doi:10.1002/mrm.25508 
Bester, M., Forkert, N.D., Stellmann, J.P., Aly, L., Drabik, A., Young, K.L., Heesen, C., Fiehler, J., Siemonsen, S., 2015. Increased perfusion in normal appearing white matter in high inflammatory multiple sclerosis patients. PLoS One 10,1-12. doi:10.1371/journal.pone.0119356

Beutler, E., West, C., 2017. Hematologic differences between African-Americans and whites : the roles of iron deficiency and alpha-thalassemia on hemoglobin levels and mean corpuscular volume 106, 740-746. doi:10.1182/blood-2005-02-0713.Supported

Bhogal, A.A., Siero, J.C.W., Fisher, J.A., Froeling, M., Luijten, P., Philippens, M., Hoogduin, $\mathrm{H} ., 2014$. Investigating the non-linearity of the BOLD cerebrovascular reactivity response to targeted hypo/hypercapnia at 7T. Neuroimage 98, 296-305. doi:10.1016/j.neuroimage.2014.05.006

Billett, H.H., 1990. Hemoglobin and Hematocrit, Clinical Methods: The History, Physical, and Laboratory Examinations.

Blockley, N.P., Driver, I.D., Fisher, J.A., Francis, S.T., Gowland, P.A., 2012a. Measuring venous blood volume changes during activation using hyperoxia. Neuroimage 59, 3266-3274.

Blockley, N.P., Griffeth, V.E.M., Buxton, R.B., 2012b. A general analysis of calibrated BOLD methodology for measuring CMRO2 responses: comparison of a new approach with existing methods. Neuroimage 60, 279-89. doi:10.1016/j.neuroimage.2011.11.081

Blockley, N.P., Griffeth, V.E.M., Germuska, M.A., Bulte, D.P., Buxton, R.B., 2013. An analysis of the use of hyperoxia for measuring venous cerebral blood volume: comparison of the existing method with a new analysis approach. Neuroimage 72, 3340. doi:10.1016/j.neuroimage.2013.01.039

Blockley, N.P., Griffeth, V.E.M., Simon, A.B., Dubowitz, D.J., Buxton, R.B., 2015a. Calibrating the BOLD response without administering gases: Comparison of hypercapnia calibration with calibration using an asymmetric spin echo. Neuroimage 104, 423-429. doi:10.1016/j.neuroimage.2014.09.061

Blockley, N.P., Griffeth, V.E.M., Stone, A.J., Hare, H. V., Bulte, D.P., 2015b. Sources of systematic error in calibrated BOLD based mapping of baseline oxygen extraction fraction. Neuroimage 122, 105-113. doi:10.1016/j.neuroimage.2015.07.059

Blockley, N.P., Harkin, J.W., Bulte, D.P., 2017. Rapid cerebrovascular reactivity mapping: Enabling vascular reactivity information to be routinely acquired. Neuroimage 159, 214223. doi:10.1016/j.neuroimage.2017.07.048

Boxerman, J.L., Hamberg, L.M., Rosen, B.R., Weisskoff, R.M., 1995. Mr contrast due to intravascular magnetic susceptibility perturbations. Magn. Reson. Med. 34, 555-566. doi:10.1002/mrm.1910340412

Broom, K.A., Anthony, D.C., Blamire, A.M., Waters, S., Styles, P., Perry, V.H., Sibson, N.R., 2005. MRI reveals that early changes in cerebral blood volume precede blood-brain barrier breakdown and overt pathology in MS-like lesions in rat brain. J Cereb Blood Flow Metab 25, 204-216. doi:9600020 [pii]/n10.1038/sj.jcbfm.9600020

Bulte, D.P., Chiarelli, P.A., Wise, R.G., Jezzard, P., 2007a. Cerebral perfusion response to hyperoxia. J. Cereb. Blood Flow Metab. 27, 69-75.

Bulte, D.P., Chiarelli, P., Wise, R., Jezzard, P., 2007b. Measurement of cerebral blood volume in humans using hyperoxic MRI contrast. J. Magn. Reson. Imaging 26, 894899. doi:10.1002/jmri.21096

Bulte, D.P., Kelly, M., Germuska, M., Xie, J., Chappell, M.A., Okell, T.W., Bright, M.G., Jezzard, P., 2012. Quantitative measurement of cerebral physiology using respiratorycalibrated MRI. Neuroimage 60, 582-591. doi:10.1016/j.neuroimage.2011.12.017

Buxton, R.B., Frank, L.R., Wong, E.C., Siewert, B., Warach, S., Edelman, R.R., 1998. A general kinetic model for quantitative perfusion imaging with arterial spin labeling. Magn. Reson. Med. 40, 383-96.

Buxton, R.B., Wong, E.C., Frank, L.R., 1998. Dynamics of blood flow and oxygenation changes during brain activation: the balloon model. Magn. Reson. Med. 39, 855-864.

Calamante, F., Osch, M.J.P. Van, Knutsson, L., 2016. A novel approach to measure local cerebral haematocrit using MRI. doi:10.1177/0271678X15606143 
Carrera, E., Jones, P.S., Iglesias, S., Guadagno, J. V, Warburton, E.A., Fryer, T.D., Aigbirhio, F.I., Baron, J.-C., 2011. The Vascular Mean Transit Time: A Surrogate for the Penumbra Flow Threshold? J. Cereb. Blood Flow Metab. 31, 1027-1035. doi:10.1038/jcbfm.2010.197

Chen, J.J., Pike, G.B., 2010. MRI measurement of the BOLD-specific flow-volume relationship during hypercapnia and hypocapnia in humans. Neuroimage 53, 383-391. doi:10.1016/j.neuroimage.2010.07.003

Chen, J.J., Pike, G.B., 2010. Global cerebral oxidative metabolism during hypercapnia and hypocapnia in humans: implications for BOLD fMRI. J. Cereb. Blood Flow Metab. 30, 1094-1099.

Chen, J.J., Pike, G.B., 2009. BOLD-specific cerebral blood volume and blood flow changes during neuronal activation in humans. NMR Biomed. 22, n/a-n/a. doi:10.1002/nbm.1411

Chen, Z., Zhang, X., Yuan, C., Zhao, X., van Osch, M.J.P., 2017. Measuring the labeling efficiency of pseudocontinuous arterial spin labeling. Magn. Reson. Med. 77, 18411852. doi:10.1002/mrm.26266

Cheng, C., Chan, J., Cembrowski, G., van Assendelft, O., 2004. Complete blood count reference interval diagrams derived from NHANES III: stratification by age, sex, and race. Lab. Hematol. 10, 42-53.

Chiarelli, P.A., Bulte, D.P., Wise, R., Gallichan, D., Jezzard, P., 2007. A calibration method for quantitative BOLD fMRI based on hyperoxia. Neuroimage 37, 808-820.

Clement, P., Mutsaerts, H.-J., Václavů, L., Ghariq, E., Pizzini, F.B., Smits, M., Acou, M., Jovicich, J., Vanninen, R., Kononen, M., Wiest, R., Rostrup, E., Bastos-Leite, A.J., Larsson, E.-M., Achten, E., 2017. Variability of physiological brain perfusion in healthy subjects - A systematic review of modifiers. Considerations for multi-center ASL studies. J. Cereb. Blood Flow Metab. 271678X17702156. doi:10.1177/0271678X17702156

Croal, P.L., Driver, I.D., Francis, S.T., Gowland, P.A., 2017a. Field strength dependence of grey matter R2* on venous oxygenation. Neuroimage 146, 327-332. doi:10.1016/J.NEUROIMAGE.2016.10.004

Croal, P.L., Hall, E.L., Driver, I.D., Brookes, M.J., Gowland, P.A., Francis, S.T., 2015. The effect of isocapnic hyperoxia on neurophysiology as measured with MRI and MEG. Neuroimage 105, 323-31. doi:10.1016/j.neuroimage.2014.10.036

Croal, P.L., Leung, J., Kosinski, P.D., Shroff, M., Odame, I., Kassner, A., 2017b. Assessment of cerebral blood flow with magnetic resonance imaging in children with sickle cell disease : A quantitative ... Assessment of cerebral blood flow with magnetic resonance imaging in children with sickle cell disease : A quantitative comparison. Brain Behav. 7, e00811. doi:10.1002/brb3.811

Cunningham, A.S., Salvador, R., Coles, J.P., Chatfield, D.A., Bradley, P.G., Johnston, A.J., Steiner, L.A., Fryer, T.D., Aigbirhio, F.I., Smielewski, P., Williams, G.B., Carpenter, T.A., Gillard, J.H., Pickard, J.D., Menon, D.K., 2005. Physiological thresholds for irreversible tissue damage in contusional regions following traumatic brain injury. Brain 128, 19311942. doi:10.1093/brain/awh536

Dai, W., Garcia, D., de Bazelaire, C., Alsop, D.C., 2008. Continuous flow-driven inversion for arterial spin labeling using pulsed radio frequency and gradient fields. Magn. Reson. Med. 60, 1488-1497. doi:10.1002/mrm.21790

Daniel, W.A., 1973. Hematocrit: maturity relationship in adolescence. Pediatrics 52, 388-94.

Davis, T.L., Kwong, K.K., Weisskoff, R.M., Rosen, B.R., 1998. Calibrated functional MRI: Mapping the dynamics of oxidative metabolism. Proc. Natl. Acad. Sci. 95, 1834-1839. doi:10.1073/pnas.95.4.1834

De Vis, J.B., Petersen, E.T., Bhogal, A., Hartkamp, N.S., Klijn, C.J., Kappelle, L., Hendrikse, J., 2015. Calibrated MRI to Evaluate Cerebral Hemodynamics in Patients with an Internal Carotid Artery Occlusion. J. Cereb. Blood Flow Metab. 35, 1015-1023. doi:10.1038/jcbfm.2015.14

Derdeyn, C.P., Videen, T.O., Yundt, K.D., Fritsch, S.M., Carpenter, D.A., Grubb, R.L., Powers, W.J., 2002. Variability of cerebral blood volume and oxygen extraction: stages 
of cerebral haemodynamic impairment revisited. Brain 125, 595-607.

doi:10.1093/brain/awf047

Diedler, J., Sykora, M., Rupp, A., Poli, S., Karpel-Massler, G., Sakowitz, O., Steiner, T., 2009. Impaired Cerebral Vasomotor Activity in Spontaneous Intracerebral Hemorrhage. Stroke 40, 815-819. doi:10.1161/STROKEAHA.108.531020

Domsch, S., Mürle, B., Weingärtner, S., Zapp, J., Wenz, F., Schad, L.R., 2017. Oxygen extraction fraction mapping at 3 Tesla using an artificial neural network: A feasibility study. Magn. Reson. Med. doi:10.1002/mrm.26749

Donahue, M.J., Achten, E., Cogswell, P.M., De Leeuw, F.-E., Derdeyn, C.P., Dijkhuizen, R.M., Fan, A.P., Ghaznawi, R., Heit, J.J., Ikram, M.A., Jezzard, P., Jordan, L.C., Jouvent, E., Knutsson, L., Leigh, R., Liebeskind, D.S., Lin, W., Okell, T.W., Qureshi, A.I., Stagg, C.J., van Osch, M.J., van Zijl, P.C., Watchmaker, J.M., Wintermark, M., Wu, O., Zaharchuk, G., Zhou, J., Hendrikse, J., 2017. Consensus statement on current and emerging methods for the diagnosis and evaluation of cerebrovascular disease. J. Cereb. Blood Flow Metab. 0271678X1772183. doi:10.1177/0271678X17721830

Douglas, C.G., Haldane, J.S., 1905. The Regulation of Normal Breathing. J. Physiol. 38, $420-440$.

Driver, I., Blockley, N.P., Fisher, J., Francis, S., Gowland, P., 2010. The change in cerebrovascular reactivity between $3 \mathrm{~T}$ and $7 \mathrm{~T}$ measured using graded hypercapnia. Neuroimage 51, 274-9. doi:10.1016/j.neuroimage.2009.12.113

Driver, I.D., Wharton, S.J., Croal, P.L., Bowtell, R., Francis, S.T., Gowland, P.A., 2014. Global intravascular and local hyperoxia contrast phase-based blood oxygenation measurements. Neuroimage 101, 458-65. doi:10.1016/j.neuroimage.2014.07.050

Driver, I.D., Whittaker, J.R., Bright, M.G., Muthukumaraswamy, S.D., Murphy, K., 2016. Arterial CO2 Fluctuations Modulate Neuronal Rhythmicity: Implications for MEG and fMRI Studies of Resting-State Networks. J. Neurosci. 36, 8541-50. doi:10.1523/JNEUROSCI.4263-15.2016

Driver, I.D., Wise, R.G., Murphy, K., 2017. Graded hypercapnia-calibrated BOLD: Beyond the Iso-metabolic hypercapnic assumption. Front. Neurosci. 11, 1-9. doi:10.3389/fnins.2017.00276

Du, F., Zhu, X.H., Zhang, Y., Friedman, M., Zhang, N., U urbil, K., Chen, W., 2008. Tightly coupled brain activity and cerebral ATP metabolic rate. Proc. Natl. Acad. Sci. 105, 6409-6414.

Ellika, S.K., Jain, R., Patel, S.C., Scarpace, L., Schultz, L.R., Rock, J.P., Mikkelsen, T., 2007. Role of perfusion CT in glioma grading and comparison with conventional MR imaging features. Am. J. Neuroradiol. 28, 1981-1987. doi:10.3174/ajnr.A0688

Engvall, C., Ryding, E., Wirestam, R., Holtås, S., Ljunggren, K., Ohlsson, T., Reinstrup, P., 2008. Human cerebral blood volume (CBV) measured by dynamic susceptibility contrast MRI and 99mTc-RBC SPECT. J. Neurosurg. Anesthesiol. 20, 41-4. doi:10.1097/ANA.0b013e31815d4c70

Fan, A.P., Jahanian, H., Holdsworth, S.J., Zaharchuk, G., 2016. Comparison of cerebral blood flow measurement with [15O]-water positron emission tomography and arterial spin labeling magnetic resonance imaging: A systematic review. J. Cereb. Blood Flow Metab. 36, 842-61. doi:10.1177/0271678X16636393

Faraco, C.C., Strother, M.K., Dethrage, L.M., Jordan, L., Singer, R., Clemmons, P.F., Donahue, M.J., 2015a. Dual echo vessel-encoded ASL for simultaneous BOLD and CBF reactivity assessment in patients with ischemic cerebrovascular disease. Magn. Reson. Med. 73, 1579-92. doi:10.1002/mrm.25268

Faraco, C.C., Strother, M.K., Siero, J.C., Arteaga, D.F., Scott, A.O., Jordan, L.C., Donahue, M.J., 2015b. The cumulative influence of hyperoxia and hypercapnia on blood oxygenation and R2*. J. Cereb. Blood Flow Metab. 35, 2032-2042. doi:10.1038/jcbfm.2015.168

Fisher, J.A., 2016. The CO2 stimulus for cerebrovascular reactivity: Fixing inspired concentrations vs. targeting end-tidal partial pressures. J. Cereb. Blood Flow Metab. 36, 1004-1011. doi:10.1177/0271678X16639326 
Floyd, T.F., Clark, J.M., Gelfand, R., Detre, J.A., Ratcliffe, S., Guvakov, D., Lambertsen, C.J., Eckenhoff, R.G., 2003. Independent cerebral vasoconstrictive effects of hyperoxia and accompanying arterial hypocapnia at 1 ATA. J. Appl. Physiol. 95, 2453-2461.

Fox, P.T., Raichle, M.E., 1986. Focal physiological uncoupling of cerebral blood flow and oxidative metabolism during somatosensory stimulation in human subjects. Proc. Natl. Acad. Sci. 83, 1140-1144.

Fujita, N., Matsumoto, K., Tanaka, H., Watanabe, Y., Murase, K., 2006. Quantitative study of changes in oxidative metabolism during visual stimulation using absolute relaxation rates. NMR Biomed. 19, 60-8. doi:10.1002/nbm.1001

Gao, Y.-Z., Zhang, J.-J., Liu, H., Wu, G.-Y., Xiong, L., Shu, M., 2013. Regional cerebral blood flow and cerebrovascular reactivity in Alzheimer's disease and vascular dementia assessed by arterial spinlabeling magnetic resonance imaging. Curr. Neurovasc. Res. 10, 49-53.

Gauthier, C.J., Hoge, R.D., 2012a. A generalized procedure for calibrated MRI incorporating hyperoxia and hypercapnia. Hum. Brain Mapp. 60, 1212-1225.

Gauthier, C.J., Hoge, R.D., 2012b. Magnetic resonance imaging of resting OEF and $\mathrm{CMRO}_{2}$ using a generalized calibration model for hypercapnia and hyperoxia. Neuroimage 60, 1212-25. doi:10.1016/j.neuroimage.2011.12.056

Ge, Y., Law, M., Johnson, G., Herbert, J., Babb, J.S., Mannon, L.J., Grossman, R.I., 2005. Dynamic susceptibility contrast perfusion MR imaging of multiple sclerosis lesions: characterizing hemodynamic impairment and inflammatory activity. AJNR Am J Neuroradiol 26, 1539-1547. doi:26/6/1539 [pii]

Ge, Y., Zhang, Z., Lu, H., Tang, L., Jaggi, H., Herbert, J., Babb, J.S., Rusinek, H., Grossman, R.I., 2012. Characterizing Brain Oxygen Metabolism in Patients with Multiple Sclerosis with T2 -Relaxation-Under-Spin-Tagging MRI. J. Cereb. Blood Flow Metab. 32, 403-412. doi:10.1038/jcbfm.2011.191

Goodnough, L., Dubois, R., Nissenson, A., 2003. Not Just an Innocent Bystander? Arch. Intern. Med. 163, 1400-1405.

Griffeth, V.E.M., Blockley, N.P., Simon, A.B., Buxton, R.B., 2013. A New Functional MRI Approach for Investigating Modulations of Brain Oxygen Metabolism. PLoS One 8, 113. doi:10.1371/journal.pone.0068122

Griffeth, V.E.M., Buxton, R.B., 2011. A theoretical framework for estimating cerebral oxygen metabolism changes using the calibrated-BOLD method: modeling the effects of blood volume distribution, hematocrit, oxygen extraction fraction, and tissue signal properties on the BOLD signal. Neuroimage 58, 198-212. doi:10.1016/j.neuroimage.2011.05.077

Groopman, J.E., Itri, L.M., 1999. Chemotherapy-Induced Anemia in Adults: Incidence and Treatment. J. Natl. Cancer Inst. 91, 1616-1634.

Grubb, R.L., Raichle, M.E., Eichling, J.O., Ter-Pogossian, M.M., 1974. The effects of changes in $\mathrm{PaCO} 2$ on cerebral blood volume, blood flow, and vascular mean transit time. Stroke 5, 630-9. doi:10.1161/01.STR.5.5.630

Hall, E.L., Driver, I.D., Croal, P.L., Francis, S.T., Gowland, P.A., Morris, P.G., Brookes, M.J., 2011. The effect of hypercapnia on resting and stimulus induced MEG signals. Neuroimage 58, 1034-43. doi:10.1016/j.neuroimage.2011.06.073

Hare, H. V., Blockley, N.P., Gardener, A.G., Clare, S., Bulte, D.P., 2015. Investigating the field-dependence of the Davis model: Calibrated fMRI at 1.5, 3 and 7T. Neuroimage 112, 189-196. doi:10.1016/j.neuroimage.2015.02.068

Hare, H. V, Germuska, M., Kelly, M.E., Bulte, D.P., 2013. Comparison of CO2 in air versus carbogen for the measurement of cerebrovascular reactivity with magnetic resonance imaging. J. Cereb. Blood Flow Metab. 33, 1799-805. doi:10.1038/jcbfm.2013.131

He, X., Yablonskiy, D.A., 2007. Quantitative BOLD: Mapping of human cerebral deoxygenated blood volume and oxygen extraction fraction: Default state. Magn. Reson. Med. 57, 115-126. doi:10.1002/mrm.21108

Hoffman, W.E., Miletich, D.J., Albrecht, R.F., 1982. Cerebrovascular and cerebral metabolic responses of aged rats to changes in arterial PCO2. Neurobiol. Aging 3, 141-143. doi:10.1016/0197-4580(82)90010-0 
Hoge, R.D., Atkinson, J., Gill, B., Crelier, G.R., Marrett, S., Pike, G.B., 1999. Investigation of BOLD signal dependence on cerebral blood flow and oxygen consumption: the deoxyhemoglobin dilution model. Magn. Reson. Med. 42, 849-863.

Horvath, I., Sandor, N.T., Ruttner, Z., McLaughlin, A.C., 1994. Role of Nitric Oxide in Regulating Cerebrocortical Oxygen Consumption and Blood Flow during Hypercapnia. J. Cereb. Blood Flow Metab. 14, 503-509. doi:10.1038/jcbfm.1994.62

Hubbard, N.A., Turner, M.P., Ouyang, M., Himes, L., Thomas, B.P., Hutchison, J.L., Faghihahmadabadi, S., Davis, S.L., Strain, J.F., Spence, J., Krawczyk, D.C., Huang, H., Lu, H., Hart, J., Frohman, T.C., Frohman, E.M., Okuda, D.T., Rypma, B., 2017. Calibrated imaging reveals altered grey matter metabolism related to white matter microstructure and symptom severity in multiple sclerosis. Hum. Brain Mapp. 5390, 5375-5390. doi:10.1002/hbm.23727

Huber, L., Uludağ, K., Möller, H.E., 2017. Non-BOLD contrast for laminar fMRI in humans: CBF, CBV, and CMRO2. Neuroimage. doi:10.1016/j.neuroimage.2017.07.041

Hyder, D.S.F., 2010. Neurovascular and neurometabolic couplings in dynamic calibrated fMRI: transient oxidative neuroenergetics for block-design and event-related paradigms. Front. Neuroenergetics 2, 1-11. doi:10.3389/fnene.2010.00018

Isozaki, M., Arai, Y., Kudo, T., Kiyono, Y., Kobayashi, M., Kubota, T., Kikuta, K.I., Okazawa, H., 2010. Clinical implication and prognosis of normal baseline cerebral blood flow with impaired vascular reserve in patients with major cerebral artery occlusive disease. Ann. Nucl. Med. 24, 371-377. doi:10.1007/s12149-010-0367-9

Ito, H., Ibaraki, M., Kanno, I., Fukuda, H., Miura, S., 2005. Changes in the Arterial Fraction of Human Cerebral Blood Volume during Hypercapnia and Hypocapnia Measured by Positron Emission Tomography. J. Cereb. Blood Flow Metab. 25, 852-857. doi:10.1038/sj.jcbfm.9600076

Ito, S., Mardimae, A., Han, J., Duffin, J., Wells, G., Fedorko, L., Minkovich, L., Katznelson, R., Meineri, M., Arenovich, T., Kessler, C., Fisher, J.A., 2008. Non-invasive prospective targeting of arterial $P$ CO 2 in subjects at rest. J. Physiol. 586, 3675-3682. doi:10.1113/jphysiol.2008.154716

Jahanian, H., Noll, D.C., Hernandez-Garcia, L., 2011. B0 field inhomogeneity considerations in pseudo-continuous arterial spin labeling (pCASL): effects on tagging efficiency and correction strategy. NMR Biomed. 24, 1202-9. doi:10.1002/nbm.1675

Jain, V., Langham, M.C., Floyd, T.F., Jain, G., Magland, J.F., Wehrli, F.W., 2011. Rapid magnetic resonance measurement of global cerebral metabolic rate of oxygen consumption in humans during rest and hypercapnia. J. Cereb. Blood Flow Metab. 31, 1504-1512. doi:10.1038/jcbfm.2011.34

Jones, M., Berwick, J., Hewson-Stoate, N., Gias, C., Mayhew, J., 2005. The effect of hypercapnia on the neural and hemodynamic responses to somatosensory stimulation. Neuroimage 27, 609-623.

Jordan, J.D., Powers, W.J., 2012. Cerebral Autoregulation and Acute Ischemic Stroke. Am. J. Hypertens. 25, 946-950. doi:10.1038/ajh.2012.53

Joseph, B., Haider, A., Rhee, P., 2016. Non-invasive hemoglobin monitoring. Int. J. Surg. 33, 254-257. doi:10.1016/j.ijsu.2015.11.048

Juttukonda, M.R., Jordan, L.C., Gindville, M.C., Davis, L.T., Watchmaker, J.M., Pruthi, S., Donahue, M.J., 2017. Cerebral hemodynamics and pseudo-continuous arterial spin labeling considerations in adults with sickle cell anemia. NMR Biomed. 30, e3681. doi:10.1002/nbm.3681

Kaskinoro, K., Maksimow, A., Laitio, R., Scheinin, H., Jääskeläinen, S., 2010. Normobaric hyperoxia does not induce significant electroencephalogram changes in healthy male subjects: 7AP4-5. Eur. J. Anaesthesiol. 27, 121-122.

Kennan, R.P., Zhong, J., Gore, J.C., 1994. Intravascular susceptibility contrast mechanisms in tissues. Magn. Reson. Med. 31, 9-21. doi:10.1002/mrm.1910310103

Kettunen, M.I., Gröhn, O.H.J., Silvennoinen, M.J., Penttonen, M., Kauppinen, R.A., 2002. Effects of intracellular $\mathrm{pH}$, blood, and tissue oxygen tension on $\mathrm{T} 1 \mathrm{\rho}$ relaxation in rat brain. Magn. Reson. Med. 48, 470-477. doi:10.1002/mrm.10233 
Kety, S.S., Schmidt, C.F., 1948. The nitrous oxide method for the quantitative determination of cerebral blood flow in man: theory, procedure and normal values. J. Clin. Invest. 27, $476-483$.

Kida, I., Kennan, R.P., Rothman, D.L., Behar, K.L., Hyder, F., 2000. High-Resolution CMR O2 Mapping in Rat Cortex: A Multiparametric Approach to Calibration of BOLD Image Contrast at 7 Tesla. J. Cereb. Blood Flow Metab. 20, 847-860. doi:10.1097/00004647200005000-00012

Kolbitsch, C., Lorenz, I.H., Hörmann, C., Hinteregger, M., Löckinger, A., Moser, P.L., Kremser, C., Schocke, M., Felber, S., Pfeiffer, K.P., 2002. The influence of hyperoxia on regional cerebral blood flow ( $\mathrm{rCBF}$ ), regional cerebral blood volume ( $\mathrm{rCBV}$ ) and cerebral blood flow velocity in the middle cerebral artery (CBFVMCA) in human volunteers. Magn. Reson. Imaging 20, 535-541.

Kolbitsch, C., Lorenz, I.H., Hörmann, C., Kremser, C., Schocke, M., Felber, S., Moser, P.L., Hinteregger, M., Pfeiffer, K.P., Benzer, A., 2001. Sevoflurane and nitrous oxide increase regional cerebral blood flow (rCBF) and regional cerebral blood volume (rCBV) in a drug-specific manner in human volunteers. Magn. Reson. Imaging 19, 1253-1260. doi:10.1016/S0730-725X(01)00465-9

Koziak, A.M., Winter, J., Lee, T.-Y., Thompson, R.T., St Lawrence, K.S., 2008. Validation study of a pulsed arterial spin labeling technique by comparison to perfusion computed tomography. Magn. Reson. Imaging 26, 543-53. doi:10.1016/j.mri.2007.10.005

Kuroda, S., Shiga, T., Ishikawa, T., Houkin, K., Narita, T., Katoh, C., Tamaki, N., Iwasaki, Y., 2004. Reduced blood flow and preserved vasoreactivity characterize oxygen hypometabolism due to incomplete infarction in occlusive carotid artery diseases. J. Nucl. Med. 45, 943-9.

Kwong, K., Belliveau, J., Chesler, D., Goldberg, I., Weisskoff, R., Poncelet, B., Kennedy, D., Hoppel, B., Cohen, M., Turner, R., 1992. Dynamic magnetic resonance imaging of human brain activity during primary sensory stimulation. Proc Natl Acad Sci U S A 89, 5675-5679. doi:10.1073/pnas.89.12.5675

Lacalle-Aurioles, M., Mateos-Pérez, J.M., Guzmán-De-Villoria, J.A., Olazarán, J., CruzOrduña, I., Alemán-Gómez, Y., Martino, M.-E., Desco, M., 2014. Cerebral Blood Flow is an Earlier Indicator of Perfusion Abnormalities than Cerebral Blood Volume in Alzheimer's Disease. J. Cereb. Blood Flow Metab. 34, 654-659. doi:10.1038/jcbfm.2013.241

Lajoie, I., Nugent, S., Debacker, C., Dyson, K., Tancredi, F.B., Badhwar, A.P., Belleville, S., Deschaintre, Y., Bellec, P., Doyon, J., Bocti, C., Gauthier, S., Arnold, D., Kergoat, M.J., Chertkow, H., Monchi, O., Hoge, R.D., 2017. Application of calibrated fMRI in Alzheimer's disease. Neurolmage Clin. 15, 348-358. doi:10.1016/j.nicl.2017.05.009

Lajoie, I., Tancredi, F.B., Hoge, R.D., 2016. Regional Reproducibility of BOLD Calibration Parameter M, OEF and Resting-State CMRO2 Measurements with QUO2 MRI. PLoS One 11, e0163071. doi:10.1371/journal.pone.0163071

Lake, E.M.R., Bazzigaluppi, P., Mester, J., Thomason, L.A.M., Janik, R., Brown, M., McLaurin, J.A., Carlen, P.L., Corbett, D., Stanisz, G.J., Stefanovic, B., 2017. Neurovascular unit remodelling in the subacute stage of stroke recovery. Neuroimage 146, 869-882. doi:10.1016/j.neuroimage.2016.09.016

Lee, S.P., Duong, T.Q., Yang, G., ladecola, C., Kim, S.G., 2001. Relative changes of cerebral arterial and venous blood volumes during increased cerebral blood flow: implications for BOLD fMRI. Magn. Reson. Med. 45, 791-800.

Li, W., Liu, P., Lu, H., Strouse, J.J., Zijl, P.C.M. Van, Qin, Q., 2017. Fast Measurement of Blood T 1 in the Human Carotid Artery at 3T : Accuracy, Precision, and Reproducibility. Magn. Reson. Med. 77, 2296-2302. doi:10.1002/mrm.26325

Lin, A.-L., Fox, P.T., Yang, Y., Lu, H., Tan, L.-H., Gao, J.-H., 2008. Evaluation of MRI models in the measurement of $\mathrm{CMRO}_{2}$ and its relationship with CBF. Magn. Reson. Med. 60, 380-389. doi:10.1002/mrm.21655

Lin, W., An, H., Ford, A., Vo, K., Lee, J.-M., Zaharchuk, G., 2013. Imaging of Brain Oxygenation. In: Clinical Perfusion MRI: Techniques and Applications, in: Barker, P., X, 
G., G, Z. (Eds.), Clinical Perfusion MRI: Techniques and Applications. Cambridge University Press, pp. 75-88.

Losert, C., Peller, M., Schneider, P., Reiser, M., 2002. Oxygen enhanced MRI of the brain. Magn. Reson. Med. 48, 271-277.

Lu, H., Ge, Y., 2008. Quantitative evaluation of oxygenation in venous vessels using T2Relaxation-Under-Spin-Tagging MRI. Magn. Reson. Med. 60, 357-363. doi:10.1002/mrm.21627

Lu, H., Golay, X., Pekar, J.J., Van Zijl, P.C.M., 2003. Functional magnetic resonance imaging based on changes in vascular space occupancy. Magn. Reson. Med. 50, 263274. doi:10.1002/mrm.10519

Ludwig, H., Belle, S. Van, Barrett-lee, P., Birgega, G., Kosmidis, P., Krzakowski, M., Bokemeyer, C., Gasco, P., Nortier, J., Olmi, P., Schneider, M., Schrijvers, D., 2004. The European Cancer Anaemia Survey ( ECAS ): A large , multinational , prospective survey defining the prevalence, incidence, and treatment of anaemia in cancer patients q. Eur. J. Cancer 40, 2293-2306. doi:10.1016/j.ejca.2004.06.019

Luh, W.M., Wong, E.C., Bandettini, P.A., Hyde, J.S., 1999. QUIPSS II with thin-slice TI1 periodic saturation: a method for improving accuracy of quantitative perfusion imaging using pulsed arterial spin labeling. Magn. Reson. Med. 41, 1246-54.

Luo, F., Schmidt, K.F., Fox, G.B., Ferris, C.F., 2009. Differential responses in CBF and CBV to cocaine as measured by fMRI: Implications for pharmacological MRI signals derived oxygen metabolism assessment. J. Psychiatr. Res. 43, 1018-1024. doi:10.1016/j.jpsychires.2008.11.009

Mandell, D.M., Han, J.S., Poublanc, J., Crawley, A.P., Stainsby, J. a, Fisher, J. a, Mikulis, D.J., 2008. Mapping cerebrovascular reactivity using blood oxygen level-dependent MRI in Patients with arterial steno-occlusive disease: comparison with arterial spin labeling MRI. Stroke. 39, 2021-8. doi:10.1161/STROKEAHA.107.506709

Mandeville, J.B., Marota, J.J.A., Ayata, C., Zaharchuk, G., Moskowitz, M.A., Rosen, B.R., Weisskoff, R.M., 1999. Evidence of a cerebrovascular postarteriole windkessel with delayed compliance. J. Cereb. Blood Flow Metab. 19, 679-689.

Mansfield, P., 1977. Multi-planar image formation using NMR spin echoes. J. Phys. C Solid State Phys. 10, L55-L58. doi:10.1088/0022-3719/10/3/004

Mark, C.I., Pike, G.B., 2012. Indication of BOLD-Specific Venous Flow-Volume Changes from Precisely Controlled Hyperoxic vs. Hypercapnic Calibration. J. Cereb. Blood Flow Metab. 32, 709-719. doi:10.1038/jcbfm.2011.174

Marshall, O., Lu, H., Brisset, J.-C., Xu, F., Liu, P., Herbert, J., Grossman, R.I., Ge, Y., 2014. Impaired cerebrovascular reactivity in multiple sclerosis. JAMA Neurol. 71, 1275-81. doi:10.1001/jamaneurol.2014.1668

Mchedlishvili, G., Varazashvili, M., 1987. Hematocrit in Cerebral Capillaries and Veins Under Control and Ischemic Conditions. J. Cereb. Blood Flow Metab. 7, 739-744.

Merola, A., Germuska, M.A., Warnert, E.A., Richmond, L., Helme, D., Khot, S., Murphy, K., Rogers, P.J., Hall, J.E., Wise, R.G., 2017. Mapping the pharmacological modulation of brain oxygen metabolism: The effects of caffeine on absolute CMRO2 measured using dual calibrated fMRI. Neuroimage 155, 331-343. doi:10.1016/j.neuroimage.2017.03.028

Merola, A., Murphy, K., Stone, A.J., Germuska, M.A., Griffeth, V.E.M., Blockley, N.P., Buxton, R.B., Wise, R.G., 2016. Measurement of oxygen extraction fraction (OEF): An optimized BOLD signal model for use with hypercapnic and hyperoxic calibration. Neuroimage 129, 159-174. doi:10.1016/j.neuroimage.2016.01.021

Mezue, M., Segerdahl, A.R., Okell, T.W., Chappell, M.A., Kelly, M.E., Tracey, I., 2014. Optimization and Reliability of Multiple Postlabeling Delay Pseudo-Continuous Arterial Spin Labeling during Rest and Stimulus-Induced Functional Task Activation. J. Cereb. Blood Flow Metab. 34, 1919-1927. doi:10.1038/jcbfm.2014.163

Mitra, S., Chamney, P., Greenwood, R., Farrington, K., 2004. The Relationship Between Systemic and Whole-Body Hematocrit Is Not Constant during Ultrafiltration on Hemodialysis. J. Am. Sociey Nephrol. 15, 463-469. 
doi:10.1097/01.ASN.0000108970.48370.33

Mohtasib, R.S., Lumley, G., Goodwin, J.A., Emsley, H.C.A., Sluming, V., Parkes, L.M., 2012. Calibrated fMRI during a cognitive Stroop task reveals reduced metabolic response with increasing age. Neuroimage 59, 1143-1151. doi:10.1016/j.neuroimage.2011.07.092

Moreton, F.C., Dani, K.A., Goutcher, C., O'Hare, K., Muir, K.W., 2016. Respiratory challenge MRI: Practical aspects. Neurolmage Clin. 11, 667-677. doi:10.1016/j.nicl.2016.05.003

Müller, M., Voges, M., Piepgras, U., Schimrigk, K., 1995. Assessment of cerebral vasomotor reactivity by transcranial Doppler ultrasound and breath-holding. A comparison with acetazolamide as vasodilatory stimulus. Stroke 26, 96-100.

Muncie, H.L., Campbell, J.S., 2009. Alpha and Beta Thalassemia. Am. Fam. Physician 80, 339-334.

Murphy, K., Birn, R.M., Bandettini, P.A., 2013a. Resting-state fMRI confounds and cleanup. Neuroimage 80, 349-59. doi:10.1016/j.neuroimage.2013.04.001

Murphy, K., Harris, A., Wise, R., 2013b. Measuring the influence of hypercapnia on absolute CMRO2 in humans. Proc. Intl. Soc. Mag. Reson. Med., Salt Lake City, 213343.

Mutsaerts, H.J.M.M., van Osch, M.J.P., Zelaya, F.O., Wang, D.J.J., Nordhøy, W., Wang, Y., Wastling, S., Fernandez-Seara, M.A., Petersen, E.T., Pizzini, F.B., Fallatah, S., Hendrikse, J., Geier, O., Günther, M., Golay, X., Nederveen, A.J., Bjørnerud, A., Groote, I.R., 2015. Multi-vendor reliability of arterial spin labeling perfusion MRI using a near-identical sequence: Implications for multi-center studies. Neuroimage 113, 143152. doi:10.1016/j.neuroimage.2015.03.043

Nemoto, E.M., Yonas, H., Kuwabara, H., Pindzola, R.R., Sashin, D., Meltzer, C.C., Price, J.C., Chang, Y., Johnson, D.W., 2004. Identification of Hemodynamic Compromise by Cerebrovascular Reserve and Oxygen Extraction Fraction in Occlusive Vascular Disease. J. Cereb. Blood Flow Metab. 24, 1081-1089. doi:10.1097/01.WCB.0000125887.48838.37

Noth, U., Meadows, G.E., Kotajima, F., Deichmann, R., Corfield, D.R., Turner, R., 2006. Cerebral vascular response to hypercapnia: determination with perfusion MRI at 1.5 and 3.0 Tesla using a pulsed arterial spin labeling technique. J. Magn. Reson. Imaging 24, 1229-1235.

Ogawa, S., Lee, T.M., Kay, A.R., 1990. Brain magnetic resonance imaging with contrast dependent on blood oxygenation 87, 9868-9872.

Ogawa, S., Menon, R.S., Tank, D.W., Kim, S.G., Merkle, H., Ellermann, J.M., Ugurbil, K., 1993. Functional brain mapping by blood oxygenation level-dependent contrast magnetic resonance imaging. A comparison of signal characteristics with a biophysical model. Biophys. J. 64, 803-812.

Okazawa, H., Y, Y., Fujibayashi, Y., Yamauchi, H., Ishizu, K., S, N., Y, M., N, T., H, F., A, Y., J, K., 1996. Measurement of Regional Cerebral Plasma Pool and Hematocrit with Copper-62-Labeled HSA-DTS. J. Nucl. Med. 37, 1080-1085.

Pauling, L., Coryell, C., 1936. The Magnetic Properties and Structure of the Hemochromogens and Related Substances. Proc. Natl. Acad. Sci. U.S.A. 22, 159-163.

Paulson, O.B., Strandgaard, S., Edvinsson, L., 1990. Cerebral autoregulation. Cerebrovasc. Brain Metab. Rev. 2, 161-92.

Peebles, K., Celi, L., McGrattan, K., Murrell, C., Thomas, K., Ainslie, P.N., 2007. Human cerebrovascular and ventilatory $\mathrm{CO} 2$ reactivity to end-tidal, arterial and internal jugular vein PCO2. J. Physiol. 584, 347-57. doi:10.1113/jphysiol.2007.137075

Peng, S.-L., Ravi, H., Sheng, M., Thomas, B.P., Lu, H., 2017. Searching for a truly "isometabolic" gas challenge in physiological MRI. J. Cereb. Blood Flow Metab. 37, 715725. doi:10.1177/0271678X16638103

Poublanc, J., Han, J.S., Mandell, D.M., Conklin, J., Stainsby, J.A., Fisher, J.A., Mikulis, D.J., Crawley, A.P., 2013. Vascular steal explains early paradoxical blood oxygen leveldependent cerebrovascular response in brain regions with delayed arterial transit times. Cerebrovasc. Dis. Extra 3, 55-64. doi:10.1159/000348841

Powers, W.J., 1991. Cerebral hemodynamics in ischemic cerebrovascular disease. Ann. Neurol. 29, 231-40. doi:10.1002/ana.410290302 
Prisman, E., Slessarev, M., Han, J., Poublanc, J., Mardimae, A., Crawley, A., Fisher, J., Mikulis, D., 2008. Comparison of the effects of independently controlled end tidal PCO2 and PO2 on blood oxygen level-dependent (BOLD) MRI. J. Magn. Reson. Imaging 27, 185-191.

Qiu, M., Ramani, R., Swetye, M., Rajeevan, N., Constable, R.T., 2008. Anesthetic effects on regional CBF, BOLD, and the coupling between task-induced changes in CBF and BOLD: An fMRI study in normal human subjects. Magn. Reson. Med. 60, 987-996. doi:10.1002/mrm.21759

Rees, D.C., Williams, T.N., Gladwin, M.T., 2010. Sickle-cell disease. Lancet 376, 20182031. doi:10.1016/S0140-6736(10)61029-X

Rhodes, C.G., Lenzi, G.L., Frackowiak, R.S.J., Jones, T., Pozzilli, C., 1981. Measurement of $\mathrm{CBF}$ and CMRO2 Using the Continuous Inhalation of C15O2 and 15O2. J. Neurol. Sci. 50, 381-389.

Richiardi, J., Monsch, A.U., Haas, T., Barkhof, F., Van de Ville, D., Radü, E.W., Kressig, R.W., Haller, S., 2015. Altered cerebrovascular reactivity velocity in mild cognitive impairment and Alzheimer's disease. Neurobiol. Aging 36, 33-41. doi:10.1016/j.neurobiolaging.2014.07.020

Robbins, P.A., Conway, J., Cunningham, D.A., Khamnei, S., Paterson, D.J., 1990. A comparison of indirect methods for continuous estimation of arterial PCO2 in men. J. Appl. Physiol. 68, 1727-31.

Rodgers, G.M., Becker, P.S., Blinder, M., Cella, D., Chanan-Khan, A., Cleeland, C., Coccia, P.F., Djulbegovic, B., Gilreath, J.A., Kraut, E.H., Matulonis, U.A., Millenson, M.M., Reinke, D., Rosenthal, J., Schwartz, R.N., Soff, G., Stein, R.S., Vlahovic, G., Alva III, W., 2012. Cancer- and Induced Anemia Clinical Practice Guidelines in Oncology. Natl. Compr. Cancer Netw. 10, 628-653.

Rodgers, Z.B., Englund, E.K., Langham, M.C., Magland, J.F., Wehrli, F.W., 2015. Rapid T2and susceptometry-based CMRO2 quantification with interleaved TRUST (iTRUST). Neuroimage 106, 441-450. doi:10.1016/j.neuroimage.2014.10.061

Rossi, C., Boss, A., Donati, O.F., Luechinger, R., Kollias, S.S., Valavanis, A., Hodler, J., Nanz, D., 2012. Manipulation of cortical gray matter oxygenation by hyperoxic respiratory challenge: field dependence of $R(2){ }^{*}$ and MR signal response. NMR Biomed. 25, 1007-14. doi:10.1002/nbm.2775

Sam, K., Crawley, A.P., Conklin, J., Poublanc, J., Sobczyk, O., Mandell, D.M., Venkatraghavan, L., Duffin, J., Fisher, J.A., Black, S.E., Mikulis, D.J., 2016. Development of White Matter Hyperintensity Is Preceded by Reduced Cerebrovascular Reactivity. Ann. Neurol. 80, 277-285. doi:10.1002/ana.24712

Schmithorst, V.J., Hernandez-Garcia, L., Vannest, J., Rajagopal, A., Lee, G., Holland, S.K., 2014. Optimized simultaneous ASL and BOLD functional imaging of the whole brain. J. Magn. Reson. Imaging 39, 1104-17. doi:10.1002/jmri.24273

Schramm, W.M., Bartunek, A., Gilly, H., 1997. Effect of local limb temperature on pulse oximetry and the plethysmographic pulse wave. Int. J. Clin. Monit. Comput. 14, 17-22.

Seevinck, P.R., Deddens, L.H., Dijkhuizen, R.M., 2010. Magnetic resonance imaging of brain angiogenesis after stroke. Angiogenesis 13,101-111. doi:10.1007/s10456-0109174-0

Seo, H.J., Bahk, W.M., Jun, T.Y., Chae, J.H., 2007. The effect of oxygen inhalation on cognitive function and EEG in healthy adults. Clin. Psychopharmacol. Neurosci. 5, 2530.

Severinghaus, J.W., 1979. Simple, accurate equations for human blood O2 dissociation computations. J. Appl. Physiol. 46.

Sheng, M., Liu, P., Mao, D., Ge, Y., Lu, H., 2017a. The impact of hyperoxia on brain activity: A resting-state and task-evoked electroencephalography (EEG) study. PLoS One 12, e0176610. doi:10.1371/journal.pone.0176610

Sheng, M., Lu, H., Liu, P., Li, Y., Ravi, H., Peng, S.-L., Diaz-Arrastia, R., Devous, M.D., Womack, K.B., 2017b. Sildenafil Improves Vascular and Metabolic Function in Patients with Alzheimer's Disease. J. Alzheimers. Dis. 60, 1-14. doi:10.3233/JAD-161006 
Sicard, K.M., Duong, T.Q., 2005. Effects of hypoxia, hyperoxia, and hypercapnia on baseline and stimulus-evoked BOLD, CBF, and CMRO2 in spontaneously breathing animals. Neuroimage 25, 850-858.

Silvennoinen, M.J., Kettunen, M.I., Kauppinen, R.A., 2003. Effects of hematocrit and oxygen saturation level on blood spin-lattice relaxation. Magn. Reson. Med. 49, 568-571. doi:10.1002/mrm.10370

Slessarev, M., Han, J., Mardimae, A., Prisman, E., Preiss, D., Volgyesi, G., Ansel, C., Duffin, J., Fisher, J.A., 2007. Prospective targeting and control of end-tidal $\mathrm{CO} 2$ and $\mathrm{O} 2$ concentrations. J. Physiol. 581, 1207-1219.

Smeeing, D.P.J., Hendrikse, J., Petersen, E.T., Donahue, M.J., de Vis, J.B., 2016. Arterial Spin Labeling and Blood Oxygen Level-Dependent MRI Cerebrovascular Reactivity in Cerebrovascular Disease: A Systematic Review and Meta-Analysis. Cerebrovasc. Dis. 42, 288-307. doi:10.1159/000446081

Smith, D.B.D., Strawbridge, P.J., 1974. Auditory and Visual Evoked Potentials During Hyperoxia. Electroencephalogr. Clin. Neurophysiol. 37, 393-398.

Sobczyk, O., Battisti-Charbonney, A., Poublanc, J., Crawley, A.P., Sam, K., Fierstra, J., Mandell, D.M., Mikulis, D.J., Duffin, J., Fisher, J.A., 2014. Assessing cerebrovascular reactivity abnormality by comparison to a reference atlas. J. Cereb. Blood Flow Metab. 1-8. doi:10.1038/jcbfm.2014.184

Spano, V.R., Mandell, D.M., Poublanc, J., Sam, K., Battisti-Charbonney, A., Pucci, O., Han, J.S., Crawley, A.P., Fisher, J.A., Mikulis, D.J., 2013. CO2 Blood Oxygen Leveldependent MR Mapping of Cerebrovascular Reserve in a Clinical Population: Safety, Tolerability, and Technical Feasibility. Radiology 266, 592-598. doi:10.1148/radiol.12112795

Stefanovic, B., Pike, G.B., 2005. Venous refocusing for volume estimation: VERVE functional magnetic resonance imaging. Magn. Reson. Med. 53, 339-347. doi:10.1002/mrm.20352

Stirnberg, R., Huijbers, W., Brenner, D., Poser, B.A., Breteler, M., Stöcker, T., 2017. Rapid whole-brain resting-state fMRI at $3 \mathrm{~T}$ : Efficiency-optimized three-dimensional EPI versus repetition time-matched simultaneous-multi-slice EPI. Neuroimage 163, 81-92. doi:10.1016/j.neuroimage.2017.08.031

Stone, A.J., Blockley, N.P., 2017. A streamlined acquisition for mapping baseline brain oxygenation using quantitative BOLD. Neuroimage 147, 79-88. doi:10.1016/J.NEUROIMAGE.2016.11.057

Suri, S., Mackay, C.E., Kelly, M.E., Germuska, M., Tunbridge, E.M., Frisoni, G.B., Matthews, P.M., Ebmeier, K.P., Bulte, D.P., Filippini, N., 2015. Reduced cerebrovascular reactivity in young adults carrying the APOE $\varepsilon 4$ allele. Alzheimer's Dement. 11, 648-57. doi:10.1016/j.jalz.2014.05.1755

Swain, R.A., Harris, A.B., Wiener, E.C., Dutka, M. V., Morris, H.D., Theien, B.E., Konda, S., Engberg, K., Lauterbur, P.C., Greenough, W.T., 2003. Prolonged exercise induces angiogenesis and increases cerebral blood volume in primary motor cortex of the rat. Neuroscience 117, 1037-1046. doi:10.1016/S0306-4522(02)00664-4

Tadamura, E., Hatabu, H., Li, W., Prasad, P. V, Edelman, R.R., 1997. Effect of Oxygen Inhalation on Relaxation Times in Various Tissues. J. Magn. Reson. Imaging 7, 220 225.

Talati, P., Rane, S., Skinner, J., Gore, J., Heckers, S., 2015. Increased hippocampal blood volume and normal blood flow in schizophrenia. Psychiatry Res. 232, 219-225. doi:10.1007/s00210-015-1172-8.The

Thesen, T., Leontiev, O., Song, T., Dehghani, N., Hagler Jr, D.J., Huang, M., Buxton, R., Halgren, E., Hagler, D.J., 2012. Depression of cortical activity in humans by mild hypercapnia. Hum. Brain Mapp. 33, 715-726. doi:10.1002/hbm.21242

Thulborn, K.R., Waterton, J.C., Matthews, P.M., Radda, G.K., 1982. Oxygenation dependence of the transverse relaxation time of water protons in whole blood at high field. Biochim. Biophys. Acta 714, 265-270.

Uh, J., Lin, A.-L., Lee, K., Liu, P., Fox, P., Lu, H., 2011. Validation of VASO cerebral blood 
volume measurement with positron emission tomography. Magn. Reson. Med. 65, 744749. doi: $10.1002 / \mathrm{mrm} .22667$

van der Zwaag, W., Francis, S., Head, K., Peters, A., Gowland, P., Morris, P., Bowtell, R., 2009. fMRI at 1.5, 3 and $7 \mathrm{~T}$ : characterising BOLD signal changes. Neuroimage 47, 1425-34. doi:10.1016/j.neuroimage.2009.05.015

Van Essen, D.C., Smith, S.M., Barch, D.M., Behrens, T.E.J., Yacoub, E., Ugurbil, K., WUMinn HCP Consortium, 2013. The WU-Minn Human Connectome Project: An overview. Neuroimage 80,62-79. doi:10.1016/j.neuroimage.2013.05.041

van Iperen, C.E., van de Wiel, A., Marx, J.J.M., 2001. Acute event-related anaemia. Br. J. 739-743.

Whittaker, J.R., Bright, M.G., Driver, I.D., Murphy, K., 2016. Beat-to-beat blood pressure fluctuations are present in time-frequency dynamics of resting-state fMRI, in: ISMRM 24th Annual Meeting. Singapore, p. \#0309.

Wise, R.G., Harris, A.D., Stone, A., Murphy, K., 2013. Measurement of OEF and absolute CMRO 2: MRI-based methods using interleaved and combined hypercapnia and hyperoxia. Neuroimage 83, 135-147. doi:10.1016/j.neuroimage.2013.06.008.Measurement

Wise, R.G., Pattinson, K.T., Bulte, D.P., Chiarelli, P.A., Mayhew, S.D., Balanos, G.M., O'Connor, D.F., Pragnell, T.R., Robbins, P.A., Tracey, I., Jezzard, P., 2007. Dynamic forcing of end-tidal carbon dioxide and oxygen applied to functional magnetic resonance imaging. J Cereb Blood Flow Metab 27, 1521-1532. doi:9600465 [pii]ไr10.1038/sj.jcbfm.9600465

Wong, E.C., Buxton, R.B., Frank, L.R., 1998. Quantitative imaging of perfusion using a single subtraction (QUIPSS AND QUIPSSII). Magn. Reson. Med. 39, 702-708.

Wu, W.-C., Fernández-Seara, M., Detre, J.A., Wehrli, F.W., Wang, J., 2007. A theoretical and experimental investigation of the tagging efficiency of pseudocontinuous arterial spin labeling. Magn. Reson. Med. 58, 1020-7. doi:10.1002/mrm.21403

Wuerfel, J., Bellmann-Strobl, J., Brunecker, P., Aktas, O., McFarland, H., Villringer, A., Zipp, $F ., 2004$. Changes in cerebral perfusion precede plaque formation in multiple sclerosis: A longitudinal perfusion MRI study. Brain 127, 111-119. doi:10.1093/brain/awh007

Xu, F., Uh, J., Brier, M.R., Hart, J., Yezhuvath, U.S., Gu, H., Yang, Y., Lu, H., 2011. The influence of carbon dioxide on brain activity and metabolism in conscious humans. J. Cereb. Blood Flow Metab. 31, 58-67. doi:10.1038/jcbfm.2010.153

Yablonskiy, D.A., Haacke, E.M., 1994. Theory of NMR signal behavior in magnetically inhomogeneous tissues: the static dephasing regime. Magn. Reson. Med. 32, 749-763.

Yan, L., Liu, C.Y., Smith, R.X., Jog, M., Langham, M., Krasileva, K., Chen, Y., Ringman, J.M., Wang, D.J.J., 2016. Assessing intracranial vascular compliance using dynamic arterial spin labeling. Neuroimage 124, 433-441. doi:10.1016/j.neuroimage.2015.09.008

Young, W.L., Prohovnik, I., Ornstein, E., Ostapkovich, N., Matteo, R.S., 1991. Cerebral Blood Flow Reactivity to Changes in Carbon Dioxide Calculated Using End-Tidal versus Arterial Tensions. J. Cereb. Blood Flow Metab. 11, 1031-1035. doi:10.1038/jcbfm.1991.171

Yucel, M.A., Evans, K.C., Selb, J., Huppert, T.J., Boas, D.A., Gagnon, L., 2014. Validation of the hypercapnic calibrated fMRI method using DOT-fMRI fusion imaging. Neuroimage 102, 729-735. doi:10.1016/j.neuroimage.2014.08.052

Zappe, A.C., Uludag, K., Oeltermann, A., U urbil, K., Logothetis, N.K., 2008. The influence of moderate hypercapnia on neural activity in the anesthetized nonhuman primate. Cereb. Cortex 18, 2666-2673.

Zhang, K., Herzog, H., Mauler, J., Filss, C., Okell, T.W., Kops, E.R., Tellmann, L., Fischer, T., Brocke, B., Sturm, W., Coenen, H.H., Shah, N.J., 2014. Comparison of Cerebral Blood Flow Acquired by Simultaneous [ ${ }^{15}$ O]Water Positron Emission Tomography and Arterial Spin Labeling Magnetic Resonance Imaging. J. Cereb. Blood Flow Metab. 34, 1373-1380. doi:10.1038/jcbfm.2014.92

Zhao, J.M., Clingman, C.S., Närväinen, M.J., Kauppinen, R.A., van Zijl, P.C.M., 2007. 
Oxygenation and hematocrit dependence of transverse relaxation rates of blood at 3T. Magn. Reson. Med. 58, 592-7. doi:10.1002/mrm.21342

Zhao, L., Vidorreta, M., Soman, S., Detre, J.A., Alsop, D.C., 2016. Improving the robustness of pseudo-continuous arterial spin labeling to off-resonance and pulsatile flow velocity. Magn. Reson. Med. 1351, 1342-1351. doi:10.1002/mrm.26513

Zhou, Y., Rodgers, Z.B., Kuo, A.H., 2015. Cerebrovascular reactivity measured with arterial spin labeling and blood oxygen level dependent techniques. Magn. Reson. Imaging 33, 566-76. doi:10.1016/j.mri.2015.02.018 